\title{
Initial Boundary Value Problem of the General Three-Component Camassa-Holm Shallow Water System on an Interval
}

\author{
Lixin Tian, Qingwen Yuan, and Lizhen Wang \\ Nonlinear Scientific Research Center, Faculty of Science, Jiangsu University, Zhenjiang, Jiangsu 212013, China \\ Correspondence should be addressed to Qingwen Yuan; yuanqingwen1987@126.com
}

Received 19 July 2012; Accepted 6 November 2012

Academic Editor: Feliz Minhós

Copyright (c) 2013 Lixin Tian et al. This is an open access article distributed under the Creative Commons Attribution License, which permits unrestricted use, distribution, and reproduction in any medium, provided the original work is properly cited.

We study the initial boundary value problem of the general three-component Camassa-Holm shallow water system on an interval subject to inhomogeneous boundary conditions. First we prove a local in time existence theorem and present a weak-strong uniqueness result. Then, we establish a asymptotic stabilization of this system by a boundary feedback. Finally, we obtain a result of blow-up solution with certain initial data and boundary profiles.

\section{Introduction}

It is well known that the Camassa-Holm equation has attracted much attention in the past decade. It is a nonlinear dispersive wave equation that models the propagation of unidirectional irrotational shallow water waves over a flat bed, as well as water waves moving over an underlying shear flow. It was first introduced by Fokas and Fuchssteiner as a bi-Hamiltonian model. Cauchy problem and initial boundary value problem for Camassa-Holm equation have been studied extensively in a number of papers (see [1-15] and the references within).

$\mathrm{Fu}$ and $\mathrm{Qu}$ in [16] proposed a coupled Camassa-Holm equation,

$$
\begin{gathered}
m_{t}=2 m u_{x}+m_{x} u+(m v)_{x}+n v_{x} \\
n_{t}=2 n v_{x}+n_{x} v+(n u)_{x}+m u_{x}
\end{gathered}
$$

with $m=u-u_{x x}, n=v-v_{x x}$, which has peakon solitons in the form of a superposition of multipeakons and may as well be integrable. They investigated the local wellposedness and blow-up solutions of system (1) by means of Kato's semigroup approach to nonlinear hyperbolic evolution equation and obtained a criterion and condition on the initial data guaranteeing the development of singularities in finite time for strong solutions of system (1) by energy estimates. Recently the initial boundary value problem for the system (1) has been established in [17]; moreover, the local well-posedness and blow-up phenomena for the coupled Camassa-Holm equation were also established in [16, 18-32]. In [33], Tian and Xu obtained the compact and bounded absorbing set and the existence of the global attractor for viscous system (1) with the periodic boundary condition in by uniform prior estimate.

Recently, Fu and Qu in [34] introduced a general threecomponent Camassa-Holm equation as follows:

$$
\begin{gathered}
m_{t}=2 m u_{x}+m_{x} u+(m v+m w)_{x}+n v_{x}+l w_{x}, \\
n_{t}=2 n v_{x}+n_{x} v+(n u+n w)_{x}+m u_{x}+l w_{x}, \\
l_{t}=2 l w_{x}+l_{x} w+(l u+l v)_{x}+m u_{x}+n v_{x},
\end{gathered}
$$

where $m=u-u_{x x}, n=v-v_{x x}$, and $l=w-w_{x x}$. Equation (2) also has peakon solitons in the form of a superposition of multipeakons. Such system also conserves the $H^{1}$-norm conservation law. Moreover, the well-posedness and blowup phenomena for system (2) with peakons have been established in [35]. To our knowledge, the initial boundary value problem of (2) has not been studied yet. The first aim 
of this paper is to consider an initial boundary value problem of the following

$$
\begin{gathered}
m_{t}=2 m u_{x}+m_{x} u+(m v+m w)_{x}+n v_{x}+l w_{x}, \\
n_{t}=2 n v_{x}+n_{x} v+(n u+n w)_{x}+m u_{x}+l w_{x}, \\
l_{t}=2 l w_{x}+l_{x} w+(l u+l v)_{x}+m u_{x}+n v_{x}, \\
m(0, \cdot)=m_{0}, \quad n(0, \cdot)=n_{0}, \quad l(0, \cdot)=l_{0}, \\
m(\cdot, 0)_{\mid \Gamma_{l}}=m_{l}, \quad n(\cdot, 0)_{\mid \Gamma_{l}}=n_{l}, \quad l(\cdot, 0)_{\mid \Gamma_{l}}=l_{l}, \\
m(\cdot, 1)_{\mid \Gamma_{r}}=m_{r}, \quad n(\cdot, 1)_{\mid \Gamma_{r}}=n_{r}, \quad l(\cdot, 1)_{\mid \Gamma_{r}}=l_{r},
\end{gathered}
$$

where $\Gamma_{l}=\{t \in[0, T] \mid(u+v+w)(t, x)<0\}, \Gamma_{r}=\{t \in$ $[0, T] \mid(u+v+w)(t, x)>0\}$.

Then, we will consider the asymptotic stabilization of (3) by means of a stationary feedback law acting on the inhomogeneous boundary condition. Following the step in [11], we convert the initial boundary value problem of (3) on the interval into an ODE system and two PDE systems. Then, we can consider the system (3) easily. Consequently, we obtain a local in time existence theorem, a weak-strong uniqueness result, asymptotic stabilization result on the interval, and a result of blow-up solution, respectively.

Our paper is organized as follows. In Section 2, we will consider an initial boundary value problem and the uniqueness of the solution to (3). By using the feedback law enjoyed by (3), the asymptotic stabilization on an interval is considered in Section 3. Finally, in Section 4, a result of blowup solution with certain initial data and boundary profiles will be established.

First, we begin with a general remark that will be used many times later.

Remark 1. Let $T$ be a positive number and $\Omega_{T}=[0, T] \times$ $[0,1]$. Changing $u(t, x)$ in $-u(t, 1-x), v(t, x)$ in $-v(t, 1-$ $x), w(t, x)$ in $-w(t, 1-x)$, and $t$ in $T-t$, and it will be more convenient for us to analysis the system, if we define the following sets

$$
\begin{aligned}
& P_{l}=\{t \in[0, T] \mid(u+v+w)(t, 0)=0\}, \\
& P_{r}=\{t \in[0, T] \mid(u+v+w)(t, 1)=0\} .
\end{aligned}
$$

Let $\Lambda=\left(1-\partial_{x}^{2}\right)^{1 / 2}$, then the operator $\Lambda^{-2}$ can be expressed as

$$
\Lambda^{-2} f(x)=G * f(x)=\frac{1}{2} \int_{0}^{1} e^{-|x-y|} f(y) d y,
$$

where $G=(1 / 2) e^{-|x|}$. Now, let $A_{i}=\Lambda^{-2} B_{i}=G * B_{i}, i=1,2,3$, where $B_{i}$ is an auxiliary function which lifts the boundary values $m_{l}, m_{r}, n_{l}, n_{r}$, and $l_{l}$ defined by

$$
\begin{gathered}
B_{i}(t, x)=0, \quad(t, x) \in[0, T] \times[0,1], \\
G * B_{i}(t, 0)=v_{l_{i}}(t), \quad G * B_{i}(t, 1)=v_{r_{i}}(t), \quad \forall t \in[0, T],
\end{gathered}
$$

where $i=1,2,3$.
Setting $u=p+G * B_{1}, v=q+G * B_{2}$, and $w=r+G * B_{3}$, we can further rewrite the system (3) as

$$
\begin{gathered}
p=G * m, \quad q=G * n, \quad r=G * l, \\
p(t, 0)=p(t, 1)=0, \quad q(t, 0)=q(t, 1)=0, \\
r(t, 0)=r(t, 1)=0, \\
m_{t}=\left(p+G * B_{1}+v+w\right) m_{x} \\
+\left[2\left(p+G * B_{1}\right)_{x}+v_{x}+w_{x}\right] m+n v_{x}+l w_{x}, \\
n_{t}=\left(q+G * B_{2}+u+w\right) n_{x} \\
+\left[2\left(q+G * B_{2}\right)_{x}+u_{x}+w_{x}\right] n+m u_{x}+l w_{x}, \\
l_{t}=\left(r+G * B_{3}+u+v\right) l_{x} \\
+\left[2\left(r+G * B_{3}\right)_{x}+u_{x}+v_{x}\right] l+m u_{x}+n v_{x}, \\
m(0, \cdot)=m_{0}, \quad m(\cdot, 0)_{\mid \Gamma_{l}}=m_{l}, \quad m(\cdot, 1)_{\mid \Gamma_{r}}=m_{r}, \\
n(0, \cdot)=n_{0}, \quad n(\cdot, 0)_{\mid \Gamma_{l}}=n_{l}, \quad n(\cdot, 1)_{\mid \Gamma_{r}}=n_{r}, \\
l(0, \cdot)=l_{0}, \quad l(\cdot, 0)_{\mid \Gamma_{l}}=l_{l}, \quad l(\cdot, 1)_{\mid \Gamma_{r}}=l_{r},
\end{gathered}
$$

where functions $m_{l}, m_{r}, n_{l}, n_{r}, l_{l}$, and $l_{r}$ in $C^{0}([0,1], R)$ are the boundary values and $m_{0}, n_{0}$, and $l_{0}$ in $L^{\infty}(0,1)$ are the initial datum.

Lemma 2. We have $A_{i}=G * B_{i} \in C^{0}\left([0, T] ; C^{\infty}[0,1]\right) \in$ $C^{0}\left([0, T] ; C^{\infty}[0,1]\right)$ and $\tilde{p}, \tilde{q}, \tilde{r} \in L^{\infty}\left((0, T), C^{1,1}([0,1])\right) \cap$ $\operatorname{Lip}\left((0, T), H_{0}^{1}(0,1)\right), i=1,2,3, m, n, l \in L^{\infty}\left(\Omega_{T}\right) \cap$ $\operatorname{Lip}\left((0, T), H^{-1}(0,1)\right)$. Moreover, we also have the bounds

$$
\begin{aligned}
& \left\|A_{i}\right\|_{L^{\infty}\left((0, T) ; C^{1,1}[0,1]\right)} \\
& \leq \frac{\cosh (1)}{\sinh (1)}\left(\left\|v_{r_{i}}\right\|_{L^{\infty}(0, T)}+\left\|v_{l_{i}}\right\|_{L^{\infty}(0, T)}\right), \\
& \quad(i=1,2,3),
\end{aligned}
$$

$\|\tilde{p}\|_{L^{\infty}\left((0, T) ; C^{1,1}[0,1]\right)} \leq 2(1+\cosh (1))\|m\|_{L^{\infty}\left(\Omega_{T}\right)}$,

$\left\|\partial_{t} \tilde{p}\right\|_{L^{\infty}\left((0, T) ; H_{0}^{1}[0,1]\right)} \leq\left\|\partial_{t} m\right\|_{L^{\infty}\left((0, T) ; H^{-1}[0,1]\right)}$,

$\|\widetilde{q}\|_{L^{\infty}\left((0, T) ; C^{1,1}[0,1]\right)} \leq 2(1+\cosh (1))\|n\|_{L^{\infty}\left(\Omega_{T}\right)}$,

$\left\|\partial_{t} \tilde{q}\right\|_{L^{\infty}\left((0, T) ; H_{0}^{1}[0,1]\right)} \leq\left\|\partial_{t} n\right\|_{L^{\infty}\left((0, T) ; H^{-1}[0,1]\right)}$,

$\|\widetilde{r}\|_{L^{\infty}\left((0, T) ; C^{1,1}[0,1]\right)} \leq 2(1+\cosh (1))\|l\|_{L^{\infty}\left(\Omega_{T}\right)}$,

$\left\|\partial_{t} \widetilde{r}\right\|_{L^{\infty}\left((0, T) ; H_{0}^{1}[0,1]\right)} \leq\left\|\partial_{t} l\right\|_{L^{\infty}\left((0, T) ; H^{-1}[0,1]\right)}$. 
Proof. $A_{i}, \tilde{p}, \tilde{q}, \tilde{r},(i=1,2,3)$ can be expressed, respectively, as

$$
\begin{aligned}
& A_{i}(t, x)=G * B_{i}(t, x) \\
& =\frac{1}{\sinh (1)}\left(\sinh (x) v_{r_{i}}(t)+\sinh (1-x) v_{l_{i}}(t)\right), \\
& (i=1,2,3), \\
& \widetilde{p}(t, x)=-\int_{0}^{x} \sinh (x-\tilde{x}) m(t, \widetilde{x}) d \widetilde{x} \\
& +\frac{\sinh (x)}{\sinh (1)} \int_{0}^{1} \sinh (1-\tilde{x}) m(t, \tilde{x}) d \tilde{x}, \\
& \widetilde{q}(t, x)=-\int_{0}^{x} \sinh (x-\tilde{x}) n(t, \tilde{x}) d \tilde{x} \\
& +\frac{\sinh (x)}{\sinh (1)} \int_{0}^{1} \sinh (1-\widetilde{x}) n(t, \widetilde{x}) d \widetilde{x}, \\
& \widetilde{r}(t, x)=-\int_{0}^{x} \sinh (x-\tilde{x}) l(t, \widetilde{x}) d \widetilde{x} \\
& +\frac{\sinh (x)}{\sinh (1)} \int_{0}^{1} \sinh (1-\tilde{x}) l(t, \tilde{x}) d \tilde{x} .
\end{aligned}
$$

Estimates (9) and (10) can be easily obtained from the above expressions.

\section{Initial Boundary Value Problem}

First, we define what we mean by a weak solution to (8). Our test functions will be in the space:

$$
\begin{aligned}
\operatorname{Adm}\left(\Omega_{T}\right)=\{\varphi & \in C^{1}\left(\Omega_{T}\right) \mid \varphi(t, x)=0 \text { on }[0, T] \backslash \Gamma_{l} \\
& \left.\times\{0\} \cup[0, T] \backslash \Gamma_{r} \times\{0\} \cup\{T\} \times[0,1]\right\} .
\end{aligned}
$$

Definition 3. When $(p, q, r) \in L^{\infty}((0, T) ; \operatorname{Lip}[0,1]) \times$ $L^{\infty}((0, T) ; \operatorname{Lip}[0,1]) \times L^{\infty}((0, T) ; \operatorname{Lip}[0,1])$, the function $(m, n, l) \in L^{\infty}\left(\Omega_{T}\right) \times L^{\infty}\left(\Omega_{T}\right) \times L^{\infty}\left(\Omega_{T}\right)$ is the weak solution to (8) if for all $\varphi \in \operatorname{Adm}\left(\Omega_{T}\right)$ :

$$
\begin{gathered}
\iint_{\Omega_{T}} m\left(\varphi_{t}-(u+v+w) \varphi_{x}+\left(p_{x}+\partial_{x} G * B_{1}\right) \varphi\right) d t d x \\
=-\iint_{\Omega_{T}}\left(n v_{x}+l w_{x}\right) \varphi(t, x) d t d x-\int_{0}^{1} m_{0}(x) \varphi(0, x) d t \\
\quad+\int_{0}^{T}((u+v+w)(t, 0) \varphi(t, 0) m(t, 0) \\
\quad-(u+v+w)(t, 1) \varphi(t, 1) m(t, 1)) d t \\
\iint_{\Omega_{T}} n\left(\varphi_{t}-(u+v+w) \varphi_{x}+\left(q_{x}+\partial_{x} G * B_{2}\right) \varphi\right) d t d x
\end{gathered}
$$

$$
\begin{gathered}
=-\iint_{\Omega_{T}}\left(m u_{x}+l w_{x}\right) \varphi(t, x) d t d x-\int_{0}^{1} n_{0}(x) \varphi(0, x) d x \\
+\int_{0}^{T}((u+v+w)(t, 0) \varphi(t, 0) n(t, 0) \\
\quad-(u+v+w)(t, 1) \varphi(t, 1) n(t, 1)) d t, \\
\iint_{\Omega_{T}} l\left(\varphi_{t}-(u+v+w) \varphi_{x}+\left(r_{x}+\partial_{x} G * B_{3}\right) \varphi\right) d t d x \\
=-\iint_{\Omega_{T}}\left(m u_{x}+n v_{x}\right) \varphi(t, x) d t d x-\int_{0}^{1} l_{0}(x) \varphi(0, x) d x \\
\quad+\int_{0}^{T}((u+v+w)(t, 0) \varphi(t, 0) l(t, 0) \\
\quad-(u+v+w)(t, 1) \varphi(t, 1) l(t, 1)) d t .
\end{gathered}
$$

It is obvious that $C_{0}^{1}\left(\Omega_{T}\right) \quad \subset \operatorname{Adm}\left(\Omega_{T}\right)$; therefore, a weak solution to system (8) is also a solution to (8) in the distribution sense. And it is clear that a regular weak solution is a classical solution.

Definition 4. For $(t, x) \in \Omega_{T}$, we consider $\omega(\cdot, t, x)$ the maximal solution satisfying

$$
\begin{gathered}
\omega_{t}=-(u+v+w)(t, \omega(t, x)), \\
\omega(0, x)=x .
\end{gathered}
$$

We consider that $\omega$ is the flow of $(u(t, x), v(t, x), w(t, x))$. For $(t, x) \in \Omega_{T}, \omega(\cdot, t, x)$ is defined on a set $[e(t, x), h(t, x)]$. Here $e(t, x)$ is basically the entrance time in $\Omega_{T}$ of the characteristic curve going through $(t, x)$.

Remark 5. Obviously $e(t, x)>0$ implies that $\omega(e(t, x), t, x) \in$ $\{0,1\}$.

In the following, we consider a partition of $\Omega_{T}$, which allows us to distinguish the different influence zones in $\Omega_{T}$.

Definition 6. Let $P=\left\{(t, x) \in \Omega_{T} \mid \exists s \in[e(t, x), h(t, x)]\right.$ such that $\omega \in\{0,1\}$ and $(u+v+w)(s, \omega(s, t, x))=0\} \cup$ $\{(s, \omega(s, 0,0)) \mid$ for all $s \in[0, T]\} \cup\{(s, \omega(s, 0,1)) \mid$ for all $s \in$ $[0, T]\}$,

$$
\begin{gathered}
I=\left\{(t, x) \in \Omega_{T} \backslash p \mid e(t, x)=0\right\}, \\
L=\left\{(t, x) \in \Omega_{T} \backslash p \mid \omega(e(t, x), t, x)=0\right\}, \\
R=\left\{(t, x) \in \Omega_{T} \backslash p \mid \omega(e(t, x), t, x)=1\right\} .
\end{gathered}
$$

Those points of the set $P$ are tangent to the boundary, which are precisely the singular points of $e$ and $h$. It's obviously that the sets $P, I, L$, and $R$ constitute a partition of $\Omega_{T}$. Furthermore, if $(t, x) \in L$, then $e(t, x) \in \Gamma_{l}$, and if $(t, x) \in R$, then $e(t, x) \in \Gamma_{r}$.

Definition 7. Here, we consider the case of data $(u, v, w) \in L^{\infty}\left([0, T] ; C^{1}([0,1])\right) \times L^{\infty}\left([0, T] ; C^{1}([0,1])\right) \times$ 
$L^{\infty}\left([0, T] ; C^{1}([0,1])\right),\left(m_{l}, n_{l}, l_{l}\right) \in C_{c}^{1}\left(\Gamma_{l}\right) \times C_{c}^{1}\left(\Gamma_{l}\right) \times C_{c}^{1}\left(\Gamma_{l}\right) ;$ $\left(m_{r}, n_{r}, l_{r}\right) \in C_{c}^{1}\left(\Gamma_{r}\right) \times C_{c}^{1}\left(\Gamma_{r}\right) \times C_{c}^{1}\left(\Gamma_{r}\right),\left(m_{0}, n_{0}, l_{0}\right) \in$ $C_{c}^{1}(0,1) \times C_{c}^{1}(0,1) \times C_{c}^{1}(0,1)$. We define the functions $m, n$, and $l$ in the following way.

When $(t, x) \in P, m(t, x)=0, n(t, x)=0$, and $l(t, x)=0$, when $(t, x) \in I$,

$$
\begin{gathered}
m(t, x)=m_{0}(\omega(0, t, x)) \\
\quad \times \exp \left(\int_{0}^{t}\left[2\left(p_{x}+\partial_{x} G * B_{1}\right)+v_{x}+w_{x}\right]\right. \\
\quad \times(s, \omega(s, t, x)) d s) \\
+\int_{0}^{t}\left(n v_{x}+l w_{x}\right)(s, \omega(s, t, x)) \\
\quad \times \exp \left(\int_{s}^{t}\left[2\left(p_{x}+\partial_{x} G * B_{1}\right)+v_{x}+w_{x}\right]\right. \\
\left.\quad \times\left(s^{\prime}, \omega\left(s^{\prime}, t, x\right)\right) d s^{\prime}\right) d s,
\end{gathered}
$$

$n(t, x)=n_{0}(\omega(0, t, x))$

$$
\begin{aligned}
& \times \exp \left(\int_{0}^{t}[\right. {\left[2\left(q_{x}+\partial_{x} G * B_{2}\right)+u_{x}+w_{x}\right] } \\
&\times(s, \omega(s, t, x)) d s) \\
&+\int_{0}^{t}\left(m u_{x}+l w_{x}\right)(s, \omega(s, t, x)) \\
& \times \exp \left(\left[2\left(q_{x}+\partial_{x} G * B_{2}\right)+u_{x}+w_{x}\right]\right. \\
&\left.\times\left(s^{\prime}, \omega\left(s^{\prime}, t, x\right)\right) d s^{\prime}\right) d s,
\end{aligned}
$$

$l(t, x)=l_{0}(\omega(0, t, x))$

$$
\begin{gathered}
\times \exp \left(\int_{0}^{t}\left[2\left(r_{x}+\partial_{x} G * B_{3}\right)+u_{x}+v_{x}\right]\right. \\
\times(s, \omega(s, t, x)) d s) \\
+\int_{0}^{t}\left(m u_{x}+n v_{x}\right)(s, \omega(s, t, x)) \\
\times \exp \left(\int_{s}^{t}\left[2\left(r_{x}+\partial_{x} G * B_{3}\right)+u_{x}+v_{x}\right]\right. \\
\left.\times\left(s^{\prime}, \omega\left(s^{\prime}, t, x\right)\right) d s^{\prime}\right) d s,
\end{gathered}
$$

when $(t, x) \in L$,

$$
\begin{aligned}
m(t, x)= & m_{l}(e(t, x)) \\
& \times \exp \left(\int_{e(t, x)}^{t}\left[2\left(p_{x}+\partial_{x} G * B_{1}\right)+v_{x}+w_{x}\right]\right. \\
& \times(s, \omega(s, t, x)) d s)
\end{aligned}
$$

$$
\begin{gathered}
+\int_{e(t, x)}^{t}\left(n v_{x}+l w_{x}\right)(r, \omega(r, t, x)) \\
\times \exp \left(\int_{s}^{t}\left[2\left(p_{x}+\partial_{x} G * B_{1}\right)+v_{x}+w_{x}\right]\right. \\
\left.\times\left(s^{\prime}, \omega\left(s^{\prime}, t, x\right)\right) d s^{\prime}\right) d r
\end{gathered}
$$$$
n(t, x)=n_{l}(e(t, x))
$$$$
\times \exp \left(\int_{e(t, x)}^{t}\left[2\left(q_{x}+\partial_{x} G * B_{2}\right)+u_{x}+w_{x}\right]\right.
$$$$
\times(s, \omega(s, t, x)) d s)
$$$$
+\int_{e(t, x)}^{t}\left(m u_{x}+l w_{x}\right)(s, \omega(s, t, x))
$$$$
\times \exp \left(\int_{s}^{t}\left[2\left(q_{x}+\partial_{x} G * B_{2}\right)+u_{x}+w_{x}\right]\right.
$$$$
\left.\times\left(s^{\prime}, \omega\left(s^{\prime}, t, x\right)\right) d s^{\prime}\right) d s
$$

$$
\begin{array}{r}
l(t, x)=l_{l}(e(t, x)) \\
\quad \times \exp \left(\int_{e(t, x)}^{t}\left[2\left(r_{x}+\partial_{x} G * B_{3}\right)+u_{x}+v_{x}\right]\right. \\
\times(s, \omega(s, t, x)) d s) \\
+\int_{e(t, x)}^{t}\left(m u_{x}+n v_{x}\right)(s, \omega(s, t, x)) \\
\quad \times \exp \left(\int_{s}^{t}\left[2\left(r_{x}+\partial_{x} G * B_{3}\right)+u_{x}+v_{x}\right]\right. \\
\left.\quad \times\left(s^{\prime}, \omega\left(s^{\prime}, t, x\right)\right) d s^{\prime}\right) d s
\end{array}
$$

when $(t, x) \in R$,

$$
\begin{aligned}
& m(t, x)= m_{l}(e(t, x)) \\
& \times \exp \left(\int_{e(t, x)}^{t}\left[2\left(p_{x}+\partial_{x} G * B_{1}\right)+v_{x}+w_{x}\right]\right. \\
&\times(s, \omega(s, t, x)) d s) \\
&+ \int_{e(t, x)}^{t}\left(n v_{x}+l w_{x}\right)(r, \omega(r, t, x)) \\
& \quad \times \exp \left(\int_{s}^{t}\left[2\left(p_{x}+\partial_{x} G * B_{1}\right)+v_{x}+w_{x}\right]\right. \\
&\left.\quad \times\left(s^{\prime}, \omega\left(s^{\prime}, t, x\right)\right) d s^{\prime}\right) d r,
\end{aligned}
$$




$$
\begin{aligned}
& n(t, x)= n_{l}(e(t, x)) \\
& \times \exp \left(\int_{e(t, x)}^{t}\left[2\left(q_{x}+\partial_{x} G * B_{2}\right)+u_{x}+w_{x}\right]\right. \\
&\times(s, \omega(s, t, x)) d s) \\
&+\int_{e(t, x)}^{t}\left(m u_{x}+l w_{x}\right)(s, \omega(s, t, x)) \\
& \times \exp \left(\int_{s}^{t}\left[2\left(q_{x}+\partial_{x} G * B_{2}\right)+u_{x}+w_{x}\right]\right. \\
& l(t, x)=\left.l_{l}(e(t, x)) \quad \times\left(s^{\prime}, \omega\left(s^{\prime}, t, x\right)\right) d s^{\prime}\right) d s, \\
& \times \exp \left(\int_{e(t, x)}^{t}\left[2\left(r_{x}+\partial_{x} G * B_{3}\right)+u_{x}+v_{x}\right]\right. \\
&\times(s, \omega(s, t, x)) d s) \\
&+\int_{e(t, x)}^{t}\left(m u_{x}+n v_{x}\right)(s, \omega(s, t, x)) \\
& \times \exp \left(\int_{s}^{t}\left[2\left(r_{x}+\partial_{x} G * B_{3}\right)+u_{x}+v_{x}\right]\right. \\
&\left.\times\left(s^{\prime}, \omega\left(s^{\prime}, t, x\right)\right) d s^{\prime}\right) d s .
\end{aligned}
$$

Lemma 8. Since $(m, n, l) \in L^{\infty}\left(\Omega_{T}\right) \times L^{\infty}\left(\Omega_{T}\right) \times L^{\infty}\left(\Omega_{T}\right)$ and satisfies (8), we immediately get that $(m, n, l)$ is the weak solution of $(8)$ and $(m, n, l) \in W^{1, \infty}\left(0, T, H^{-1}(0,1)\right) \times$ $W^{1, \infty}\left(0, T, H^{-1}(0,1)\right) \times W^{1, \infty}\left(0, T, H^{-1}(0,1)\right)$. However, the functions $m, n$, and $l$ satisfy the following estimates:

$$
\begin{aligned}
\|m\|_{C^{0}\left(\Omega_{T}\right)} & \\
\leq & {\left[\max \left(\left\|m_{0}\right\|_{L^{\infty}},\left\|m_{l}\right\|_{L^{\infty}},\left\|m_{r}\right\|_{L^{\infty}}\right)+\left\|n v_{x}+l w_{x}\right\|_{L^{\infty}(0,1)} T\right] } \\
& \times e^{T\left\|2\left(p+G * B_{1}\right)_{x}+v_{x}+w_{x}\right\|_{C^{0}\left(\Omega_{T}\right)},}
\end{aligned}
$$

$\|n\|_{C^{0}\left(\Omega_{T}\right)}$

$$
\begin{aligned}
\leq & {\left[\max \left(\left\|n_{0}\right\|_{L^{\infty}},\left\|n_{\imath}\right\|_{L^{\infty}},\left\|n_{r}\right\|_{L^{\infty}}\right)+\left\|m u_{x}+l w_{x}\right\|_{L^{\infty}(0,1)} T\right] } \\
& \times e^{T\left\|2\left(q+G * B_{2}\right)_{x}+u_{x}+w_{x}\right\|_{C^{0}\left(\Omega_{T}\right)},}
\end{aligned}
$$

$\|l\|_{C^{0}\left(\Omega_{T}\right)}$

$$
\begin{aligned}
\leq & {\left[\max \left(\left\|m_{0}\right\|_{L^{\infty}},\left\|m_{l}\right\|_{L^{\infty}},\left\|m_{r}\right\|_{L^{\infty}}\right)+\left\|m u_{x}+n v_{x}\right\|_{L^{\infty}(0,1)} T\right] } \\
& \times e^{T\left\|2\left(r+G * B_{3}\right)_{x}+u_{x}+v_{x}\right\|_{C^{0}\left(\Omega_{T}\right)}},
\end{aligned}
$$

$$
\begin{aligned}
& \left\|\partial_{t} m\right\|_{C^{0}\left(\Omega_{T}\right)} \\
& \leq\left\{2 \max \left(\left\|m_{0}\right\|_{L^{\infty}},\left\|m_{l}\right\|_{L^{\infty}},\left\|m_{r}\right\|_{L^{\infty}}\right)\right. \\
& \times\left(\left\|p+G * B_{1}\right\|_{L^{\infty}((0, T) ; \operatorname{Lip}[0,1])}\right. \\
& \left.+\|v\|_{L^{\infty}((0, T) ; \operatorname{Lip}[0,1])}+\|w\|_{L^{\infty}((0, T) ; \operatorname{Lip}[0,1])}\right) \\
& +\left[\left(\|u\|_{L^{\infty}\left(\Omega_{T}\right)}+\left\|\partial_{x x}^{2} u\right\|_{L^{\infty}\left(\Omega_{T}\right)}\right)^{2}\right. \\
& +\left(\|v\|_{L^{\infty}\left(\Omega_{T}\right)}+\left\|\partial_{x x}^{2} v\right\|_{L^{\infty}\left(\Omega_{T}\right)}\right)^{2} \\
& \left.+\left(\|w\|_{L^{\infty}\left(\Omega_{T}\right)}+\left\|\partial_{x x}^{2} w\right\|_{L^{\infty}\left(\Omega_{T}\right)}\right)^{2}\right] \\
& \times\left(1+2\left(\left\|p+G * B_{1}\right\|_{L^{\infty}((0, T) ; \operatorname{Lip}[0,1])}\right.\right. \\
& \left.\left.\left.+\|v\|_{L^{\infty}((0, T) ; \operatorname{Lip}[0,1])}+\|w\|_{L^{\infty}((0, T) ; \operatorname{Lip}[0,1])}\right)\right) T\right\} \\
& \times \exp \left(2 T \left(\left\|\left(p+G * B_{1}\right)_{x}\right\|_{L^{\infty}\left(\Omega_{T}\right)}\right.\right. \\
& \left.\left.\quad+\left\|v_{x}\right\|_{L^{\infty}\left(\Omega_{T}\right)}+\left\|w_{x}\right\|_{L^{\infty}\left(\Omega_{T}\right)}\right)\right),
\end{aligned}
$$

$\left\|\partial_{t} n\right\|_{C^{0}\left(\Omega_{T}\right)}$

$$
\begin{aligned}
& \leq\{2 \max \left(\left\|n_{0}\right\|_{L^{\infty}},\left\|n_{l}\right\|_{L^{\infty}},\left\|n_{r}\right\|_{L^{\infty}}\right) \\
& \times\left(\left\|q+G * B_{2}\right\|_{L^{\infty}((0, T) ; \operatorname{Lip}[0,1])}\right. \\
&\left.+\|u\|_{L^{\infty}((0, T) ; \operatorname{Lip}[0,1])}+\|w\|_{L^{\infty}((0, T) ; \operatorname{Lip}[0,1])}\right) \\
&+ {\left[\left(\|u\|_{L^{\infty}\left(\Omega_{T}\right)}+\left\|\partial_{x x}^{2} u\right\|_{L^{\infty}\left(\Omega_{T}\right)}\right)^{2}\right.} \\
&+\left(\|v\|_{L^{\infty}\left(\Omega_{T}\right)}+\left\|\partial_{x x}^{2} v\right\|_{L^{\infty}\left(\Omega_{T}\right)}\right)^{2} \\
&\left.+\left(\|w\|_{L^{\infty}\left(\Omega_{T}\right)}+\left\|\partial_{x x}^{2} w\right\|_{L^{\infty}\left(\Omega_{T}\right)}\right)^{2}\right] \\
& \times\left(1+2\left(\left\|q+G * B_{2}\right\|_{L^{\infty}((0, T) ; \operatorname{Lip}[0,1])}\right.\right. \\
&+\|u\|_{L^{\infty}((0, T) ; \operatorname{Lip}[0,1])} \\
&\left.\left.\left.+\|w\|_{L^{\infty}((0, T) ; \operatorname{Lip}[0,1])}\right)\right) T\right\} \\
& \times \exp \left(2 T \left(\left\|\left(q+G * B_{2}\right)_{x}\right\|_{L^{\infty}\left(\Omega_{T}\right)}\right.\right. \\
&\left.\left.+\left\|p_{x}\right\|_{L^{\infty}\left(\Omega_{T}\right)}+\left\|w_{x}\right\|_{L^{\infty}\left(\Omega_{T}\right)}\right)\right)
\end{aligned}
$$

$\left\|\partial_{t} l\right\|_{C^{0}\left(\Omega_{T}\right)}$

$$
\begin{aligned}
\leq\{ & 2 \max \left(\left\|l_{0}\right\|_{L^{\infty}},\left\|l_{l}\right\|_{L^{\infty}},\left\|l_{r}\right\|_{L^{\infty}}\right) \\
& \times\left(\left\|r+G * B_{3}\right\|_{L^{\infty}((0, T) ; \operatorname{Lip}[0,1])}\right. \\
& \left.+\|u\|_{L^{\infty}((0, T) ; \operatorname{Lip}[0,1])}+\|v\|_{L^{\infty}((0, T) ; \operatorname{Lip}[0,1])}\right) \\
+ & {\left[\left(\|u\|_{L^{\infty}\left(\Omega_{T}\right)}+\left\|\partial_{x x}^{2} u\right\|_{L^{\infty}\left(\Omega_{T}\right)}\right)^{2}\right.}
\end{aligned}
$$




$$
\begin{gathered}
+\left(\|v\|_{L^{\infty}\left(\Omega_{T}\right)}+\left\|\partial_{x x}^{2} v\right\|_{L^{\infty}\left(\Omega_{T}\right)}\right)^{2} \\
\left.+\left(\|w\|_{L^{\infty}\left(\Omega_{T}\right)}+\left\|\partial_{x x}^{2} w\right\|_{L^{\infty}\left(\Omega_{T}\right)}\right)^{2}\right] \\
\times\left(1+2\left(\left\|r+G * B_{3}\right\|_{L^{\infty}((0, T) ; \operatorname{Lip}[0,1])}\right.\right. \\
+\|p\|_{L^{\infty}((0, T) ; \operatorname{Lip}[0,1])} \\
\left.\left.\left.+\|q\|_{L^{\infty}((0, T) ; \operatorname{Lip}[0,1])}\right)\right) T\right\} \\
\times \exp \left(2 T \left(\left\|\left(r+G * B_{3}\right)_{x}\right\|_{L^{\infty}\left(\Omega_{T}\right)}\right.\right. \\
\left.\left.+\left\|u_{x}\right\|_{L^{\infty}\left(\Omega_{T}\right)}+\left\|v_{x}\right\|_{L^{\infty}\left(\Omega_{T}\right)}\right)\right) .
\end{gathered}
$$

Definition 9. We can define operator $E$ and a domain for the system (8) by: for all $p, q, r \in L^{\infty}\left((0, T) ; C^{1,1}([0,1])\right) \cap$ $\operatorname{Lip}\left([0, T] ; H_{0}^{1}(0,1)\right)$,

$$
\begin{aligned}
& E(p)=\tilde{p} \in L^{\infty}\left((0, T) ; C^{1,1}([0,1])\right) \\
& \cap \operatorname{Lip}\left([0, T] ; H_{0}^{1}(0,1)\right) \\
& E(q)=\tilde{q} \in L^{\infty}\left((0, T) ; C^{1,1}([0,1])\right) \\
& \cap \operatorname{Lip}\left([0, T] ; H_{0}^{1}(0,1)\right) \\
& E(r)=\tilde{r} \in L^{\infty}\left((0, T) ; C^{1,1}([0,1])\right) \\
& \cap \operatorname{Lip}\left([0, T] ; H_{0}^{1}(0,1)\right) \\
& C_{M_{0}, M_{1}, T} \\
&=\left\{p, q, r \in L^{\infty}\left((0, T) ; C^{1,1}([0,1])\right)\right. \\
& \quad \cap \operatorname{Lip}\left([0, T] ; H_{0}^{1}(0,1)\right) \mid\|d\|_{L^{\infty}\left((0, T) ; C^{1,1}[0,1]\right)} \\
&\left.\quad \leq M_{0},\|d\|_{\operatorname{Lip}\left((0, T) ; H_{0}^{1}(0,1)\right)} \leq M_{1}\right\}
\end{aligned}
$$

where

$$
\begin{array}{r}
d(t, x)=\max (p(t, x), q(t, x), r(t, x)), \\
(t, x) \in[0, T] \times[0,1] .
\end{array}
$$

Obviously $C_{M_{0}, M_{1}, T}$ is convex and $C_{M_{0}, M_{1}, T}$ is compact with respect to the norm $\|\cdot\|_{L^{\infty}((0, T) ; \operatorname{Lip}([0,1]))}$. We will endow $C_{M_{0}, M_{1}, T}$ with the norm $\|\cdot\|_{L^{\infty}((0, T) ; \operatorname{Lip}([0,1]))}$. There exist positive numbers $M_{0}, M_{1}$, and $T$ such that $E$ maps $C_{M_{0}, M_{1}, T}$ into itself.

Theorem 10. There exists $T>0$, and $(m, n, l)$ is a weak solution of (8) with $p, q, r \in L^{\infty}\left((0, T) ; C^{1,1}([0,1])\right) \cap$ $\operatorname{Lip}\left([0, T] ; H_{0}^{1}(0,1)\right)$ and $m, n, l \in L^{\infty}\left(\Omega_{T}\right)$. Moreover, any such solution $(p, q, r)$ is in fact in $C^{0}\left([0, T] ; W^{2, P}(0,1)\right) \cap$
$C^{1}\left([0, T] ; W_{0}^{1, p}(0,1)\right)$, for all $p<+\infty$. Furthermore, the existence time of a maximal solution $T \geq \min \left(T^{*}, \widetilde{T}\right)$, with

$$
\begin{array}{r}
T^{*}=\max _{\alpha>0, \beta>0}\left(\frac{1}{6 \alpha} \ln \left(\frac{\left|\alpha-C_{1}\right|}{4(1+\cosh (1)) \alpha}\right)\right), \\
\widetilde{T}=\max _{\alpha>0, \beta>0}\left(\frac{1}{6 \alpha} \ln \left(\frac{|\beta|}{12 \alpha^{2}}\right)\right), \\
C_{1}=\max \left[\frac{\cosh (1)}{\sinh (1)}\left(\left\|v_{r_{i}}\right\|_{L^{\infty}(0, T)}+\left\|v_{l_{i}}\right\|_{L^{\infty}(0, T)}\right)\right], \\
i=1,2,3 .
\end{array}
$$

Proof. For $\widetilde{T}>0$, we consider $m_{l}, m_{r}, n_{l}, n_{r}, l_{l}$, and $l_{r}$ in $C^{0}([0, \widetilde{T}])$ such that the sets $P_{l}$ and $P_{r}$ have only a finite number of connected components.

Let $C_{0}=\max \left(\left\|m_{i}\right\|_{L^{\infty}(0,1)},\left\|n_{i}\right\|_{L^{\infty}\left(\Gamma_{l}\right)},\left\|l_{i}\right\|_{L^{\infty}\left(\Gamma_{r}\right)}\right)$, where $i=$ $0, r, l$ and

$$
\begin{array}{r}
C_{1}=\max \left[\frac{\cosh (1)}{\sinh (1)}\left(\left\|v_{r_{i}}\right\|_{L^{\infty}(0, T)}+\left\|v_{l_{i}}\right\|_{L^{\infty}(0, T)}\right)\right], \\
i=1,2,3 .
\end{array}
$$

Now, if $u, v, w \in C_{M_{0}, M_{1}, T}$ (see (21)), we have

$$
\begin{aligned}
& \left\|2\left(p+G * B_{1}\right)_{x}+v_{x}+w_{x}\right\|_{C^{\infty}\left(\Omega_{T}\right)} \\
& \leq 2\left(\left\|\partial_{x} u\right\|_{C^{\infty}\left(\Omega_{T}\right)}+\left\|\partial_{x} v\right\|_{C^{\infty}\left(\Omega_{T}\right)}+\left\|\partial_{x} w\right\|_{C^{\infty}\left(\Omega_{T}\right)}\right), \\
& \left\|2\left(q+G * B_{2}\right)_{x}+u_{x}+w_{x}\right\|_{C^{\infty}\left(\Omega_{T}\right)} \\
& \leq 2\left(\left\|\partial_{x} u\right\|_{C^{\infty}\left(\Omega_{T}\right)}+\left\|\partial_{x} v\right\|_{C^{\infty}\left(\Omega_{T}\right)}+\left\|\partial_{x} w\right\|_{C^{\infty}\left(\Omega_{T}\right)}\right), \\
& \left\|2\left(r+G * B_{3}\right)_{x}+u_{x}+v_{x}\right\|_{C^{\infty}\left(\Omega_{T}\right)} \\
& \leq 2\left(\left\|\partial_{x} u\right\|_{C^{\infty}\left(\Omega_{T}\right)}+\left\|\partial_{x} v\right\|_{C^{\infty}\left(\Omega_{T}\right)}+\left\|\partial_{x} w\right\|_{C^{\infty}\left(\Omega_{T}\right)}\right) .
\end{aligned}
$$

For all $u, v, w \in L^{\infty}\left((0, T) ; W^{2, \infty}(0,1)\right)$, we have

$$
\begin{aligned}
& \left\|\partial_{x} u\right\|_{L^{\infty}\left(\Omega_{T}\right)} \leq 2 \sqrt{\|u\|_{L^{\infty}\left(\Omega_{T}\right)}\left\|\partial_{x x}^{2} u\right\|_{L^{\infty}\left(\Omega_{T}\right)}} \\
& \leq\left(\|u\|_{L^{\infty}\left(\Omega_{T}\right)}+\left\|\partial_{x x}^{2} u\right\|_{L^{\infty}\left(\Omega_{T}\right)}\right), \\
& \left\|\partial_{x} v\right\|_{L^{\infty}\left(\Omega_{T}\right)} \leq 2 \sqrt{\|v\|_{L^{\infty}\left(\Omega_{T}\right)}\left\|\partial_{x x}^{2} v\right\|_{L^{\infty}\left(\Omega_{T}\right)}} \\
& \leq\left(\|v\|_{L^{\infty}\left(\Omega_{T}\right)}+\left\|\partial_{x x}^{2} v\right\|_{L^{\infty}\left(\Omega_{T}\right)}\right),
\end{aligned}
$$




$$
\begin{gathered}
\left\|\partial_{x} w\right\|_{L^{\infty}\left(\Omega_{T}\right)} \leq 2 \sqrt{\|w\|_{L^{\infty}\left(\Omega_{T}\right)}\left\|\partial_{x x}^{2} w\right\|_{L^{\infty}\left(\Omega_{T}\right)}} \\
\leq\left(\|w\|_{L^{\infty}\left(\Omega_{T}\right)}+\left\|\partial_{x x}^{2} w\right\|_{L^{\infty}\left(\Omega_{T}\right)}\right), \\
\left\|n v_{x}+l w_{x}\right\|_{L^{\infty}(0,1)} \\
\leq\left[\left(\|u\|_{L^{\infty}\left(\Omega_{T}\right)}+\left\|\partial_{x x}^{2} u\right\|_{L^{\infty}\left(\Omega_{T}\right)}\right)^{2}\right. \\
+\left(\|v\|_{L^{\infty}\left(\Omega_{T}\right)}+\left\|\partial_{x x}^{2} v\right\|_{L^{\infty}\left(\Omega_{T}\right)}\right)^{2} \\
\left.+\left(\|w\|_{L^{\infty}\left(\Omega_{T}\right)}+\left\|\partial_{x x}^{2} w\right\|_{L^{\infty}\left(\Omega_{T}\right)}\right)^{2}\right] \\
\left\|m u_{x}+l w_{x}\right\|_{L^{\infty}(0,1)} \\
\leq\left[\left(\|u\|_{L^{\infty}\left(\Omega_{T}\right)}+\left\|\partial_{x x}^{2} u\right\|_{L^{\infty}\left(\Omega_{T}\right)}\right)^{2}\right. \\
+\left(\|v\|_{L^{\infty}\left(\Omega_{T}\right)}+\left\|\partial_{x x}^{2} v\right\|_{L^{\infty}\left(\Omega_{T}\right)}\right)^{2} \\
\left.+\left(\|w\|_{L^{\infty}\left(\Omega_{T}\right)}+\left\|\partial_{x x}^{2} w\right\|_{L^{\infty}\left(\Omega_{T}\right)}\right)^{2}\right], \\
\left\|m u_{x}+n v_{x}\right\|_{L^{\infty}(0,1)} \\
\leq\left[\left(\|u\|_{L^{\infty}\left(\Omega_{T}\right)}+\left\|\partial_{x x}^{2} u\right\|_{L^{\infty}\left(\Omega_{T}\right)}\right)^{2}\right. \\
+\left(\|v\|_{L^{\infty}\left(\Omega_{T}\right)}+\left\|\partial_{x x}^{2} v\right\|_{L^{\infty}\left(\Omega_{T}\right)}\right)^{2} \\
\left.+\left(\|w\|_{L^{\infty}\left(\Omega_{T}\right)}+\left\|\partial_{x x}^{2} w\right\|_{L^{\infty}\left(\Omega_{T}\right)}\right)^{2}\right] .
\end{gathered}
$$

We also define that $\widetilde{d}(t, x)=\max (\widetilde{p}(t, x), \widetilde{q}(t, x), \widetilde{r}(t, x))$, $(t, x) \in \Omega_{T}$. If $p, q, r \in C_{M_{0}, M_{1}, T}$, then from Lemmas 2 and 8 , we derive that

$$
\begin{aligned}
& \|\widetilde{d}\|_{L^{\infty}\left((0, T) ; C^{1,1}[0,1]\right)} \\
& \leq 2(1+\cosh (1))\left(C_{0}+12 T^{*}\left(M_{0}+C_{1}\right)^{2}\right) \\
& \quad \times \exp \left(6 T^{*}\left(M_{0}+C_{1}\right)\right), \\
& \left\|\partial_{t} \widetilde{d}\right\|_{C^{0}\left(\Omega_{T}\right)} \\
& \leq \quad\left[6 C_{0}\left(M_{0}+C_{1}\right)+12\left(M_{0}+2 C_{1}\right)^{2}\right. \\
& \left.\quad \times\left(1+6\left(M_{0}+C_{1}\right) \widetilde{T}\right)\right] \exp \left(6 \widetilde{T}\left(M_{0}+C_{1}\right)\right) .
\end{aligned}
$$

Finally, to obtain $\widetilde{p}, \widetilde{q}, \tilde{r} \in C_{M_{0}, M_{1}, T}$, it is sufficient to show that

$$
\begin{aligned}
& 2(1+\cosh (1))\left(C_{0}+12 T^{*}\left(M_{0}+C_{1}\right)^{2}\right) \\
& \quad \times \exp \left(6 T^{*}\left(M_{0}+C_{1}\right)\right) \leq M_{0},
\end{aligned}
$$

$$
\begin{aligned}
M_{0}+ & {\left[6 C_{0}\left(M_{0}+C_{1}\right)+12\left(M_{0}+C_{1}\right)^{2}\right.} \\
& \left.\times\left(1+6 \widetilde{T}\left(M_{0}+C_{1}\right)\right)\right] \times \exp \left(6 \widetilde{T}\left(M_{0}+C_{1}\right)\right) \\
& \leq M_{1},
\end{aligned}
$$

if we have chosen $T$ and $M_{0}$; it is easy to choose $M_{1}$ to satisfy the second inequality. For the above two inequalities, we just choose $M_{0}$ and $M_{1}$ sufficiently large and then $T$ close to 0 . More precisely:

$$
\begin{gathered}
M_{0}>2(1+\cosh (1)) C_{0}, \\
T^{*} \leq \frac{1}{6\left(M_{0}+C_{1}\right)} \ln \left(\frac{M_{0}}{4(1+\cosh (1))\left(M_{0}+C_{1}\right)}\right), \\
\widetilde{T} \leq \frac{1}{6\left(M_{0}+C_{1}\right)} \ln \left(\frac{M_{1}-M_{0}}{12\left(M_{0}+C_{1}\right)^{2}}\right) .
\end{gathered}
$$

Maximizing the bound of $T$, we can get minimum existence. Then, we get the result announced, where $\alpha=M_{0}+C_{1}, \beta=$ $M_{1}-M_{0}$.

Lemma 11. The operator $E: C_{M_{0}, M_{1}, T} \rightarrow C_{M_{0}, M_{1}, T}$ is continuous with respect to $\|\cdot\|_{L^{\infty}((0, T) ; \operatorname{Lip}[0,1])}$.

Proof. The proof is omitted here; one can see a similar proof in [8, Proposition 2.4].

Now, we can apply Shauder's fixed point theorem to the operator $E$, and we get the result that there exist fixed points $p, q, r$ such that $E(p)=p, E(q)=q$, and $E(r)=r$, so we know that there exists a wake solution of (9).

$$
p, q, r \in L^{\infty}\left((0, T) ; C^{1,1}([0,1])\right) \cap \operatorname{Lip}\left([0, T] ; H_{0}^{1}(0,1)\right) .
$$

2.1. Uniqueness. We will prove the weak-strong uniqueness of weak solution of (8) in the following.

Theorem 12. Let $(p, m),(q, n),(r, l) \in L^{\infty}\left((0, T) ; C^{1,1}([0,1])\right)$ $\cap \operatorname{Lip}\left([0, T] ; H_{0}^{1}(0,1) \times L^{\infty}((0, T) ; \operatorname{Lip}([0,1]))\right.$ be the weak solution of (7)-(8), then it is unique in $L^{\infty}\left((0, T) ; C^{1,1}([0,1])\right) \times$ $L^{\infty}\left(\Omega_{T}\right)$.

Proof. Define $\Phi=m-\widetilde{m}, \Psi=n-\widetilde{n}, \Upsilon=l-\widetilde{l}$ and $P=$ $p-\widetilde{p}, Q=q-\widetilde{q}, H=r-\widetilde{r}$, then we have

$$
\begin{aligned}
& P(t, \cdot)=G * \Phi(t, \cdot), \\
& Q(t, \cdot)=G * \Psi(t, \cdot), \\
& \mathrm{H}(t, \cdot)=G * \Upsilon(t, \cdot),
\end{aligned}
$$


where $P, Q, H \quad \in \operatorname{Lip}\left([0, T] ; H_{0}^{1}(0,1)\right)$, and $(\Phi, \Psi, \Upsilon) \quad \epsilon$ $L^{\infty}\left(\Omega_{T}\right) \times L^{\infty}\left(\Omega_{T}\right) \times L^{\infty}\left(\Omega_{T}\right)$ is the unique weak solution of

$$
\begin{aligned}
\Phi_{t}= & (p+G * B+v+w) \Phi_{x} \\
& +\left[2\left(p+G * B_{1}\right)_{x}+v_{x}+w_{x}\right] \Phi \\
& +(P+Q+H) \widetilde{m}_{x}+\left(2 P_{x}+Q_{x}+H_{x}\right) \widetilde{m} \\
& +\Psi v_{x}+\widetilde{n} Q_{x}+\Upsilon w_{x}+\widetilde{l} H_{x}, \\
\Psi_{t}= & (q+G * B+u+w) \Psi_{x} \\
& +\left[2\left(q+G * B_{2}\right)_{x}+u_{x}+w_{x}\right] \Psi \\
& +(P+Q+H) \widetilde{n}_{x}+\left(P_{x}+2 Q_{x}+H_{x}\right) \widetilde{n} \\
& +\Phi u_{x}+\widetilde{m} \Phi_{x}+\Upsilon w_{x}+\widetilde{l} H_{x}, \\
\Upsilon_{t}= & (r+G * B+u+v) \Upsilon_{x} \\
& +\left[2\left(r+G * B_{3}\right)_{x}+u_{x}+v_{x}\right] \Upsilon \\
& +(P+Q+H) \widetilde{l}_{x}+\left(P_{x}+Q_{x}+2 H_{x}\right) \widetilde{l} \\
& +\Phi u_{x}+\widetilde{m} \Phi_{x}+\Psi v_{x}+\widetilde{n} Q_{x} .
\end{aligned}
$$

Let

$$
\begin{aligned}
& b_{1}=2\left(p+G * B_{1}\right)_{x}+v_{x}+w_{x}, \\
& b_{2}=2\left(q+G * B_{2}\right)_{x}+u_{x}+w_{x}, \\
& b_{3}=2\left(r+G * B_{3}\right)_{x}+u_{x}+v_{x}, \\
& f_{1}=(P+Q+H) \widetilde{m}_{x}+\left(2 P_{x}+Q_{x}+H_{x}\right) \widetilde{m} \\
&+\Psi v_{x}+\widetilde{n} Q_{x}+\Upsilon w_{x}+\widetilde{l} H_{x}, \\
& f_{2}=(P+Q+H) \widetilde{n}_{x}+\left(P_{x}+2 Q_{x}+H_{x}\right) \widetilde{n} \\
&+\Phi u_{x}+\widetilde{m} \Phi_{x}+\Upsilon w_{x}+\widetilde{l} H_{x}, \\
& f_{3}=(P+Q+H) \widetilde{l}_{x}+\left(P_{x}+Q_{x}+2 H_{x}\right) \widetilde{l} \\
&+\Phi u_{x}+\widetilde{m} \Phi_{x}+\Psi v_{x}+\widetilde{n} Q_{x},
\end{aligned}
$$

with $i_{0}=0, i_{l}=0$, and $i_{r}=0$, where $i=\Phi, \Psi, \Upsilon$.

For $(t, x) \in P$, we have $\Phi(t, x)=0, \Psi(t, x)=0$, and $\Upsilon(t, x)=0$.

Then, we get the uniqueness result.

For $(t, x) \in I$, we have

$$
\begin{aligned}
& \Phi(t, x)=\int_{0}^{t} f_{1}(s, \omega(s, t, x)) \\
& \quad \times \exp \left(\int_{r}^{t} b_{1}\left(s^{\prime}, \omega\left(s^{\prime}, t, x\right)\right) d s^{\prime}\right) d s, \\
& \Psi(t, x)=\int_{0}^{t} f_{2}(s, \omega(s, t, x)) \\
& \quad \times \exp \left(\int_{r}^{t} b_{2}\left(s^{\prime}, \omega\left(s^{\prime}, t, x\right)\right) d s^{\prime}\right) d s,
\end{aligned}
$$

$$
\begin{aligned}
\Upsilon(t, x)=\int_{0}^{t} & f_{3}(s, \omega(s, t, x)) \\
& \quad \times \exp \left(\int_{r}^{t} b_{3}\left(s^{\prime}, \omega\left(s^{\prime}, t, x\right)\right) d s^{\prime}\right) d s .
\end{aligned}
$$

For $(t, x) \in L$, we have

$$
\begin{aligned}
& \Phi(t, x)=\int_{e(t, x)}^{t} f_{1}(s, \omega(s, t, x)) \\
& \times \exp \left(\int_{r}^{t} b_{1}\left(s^{\prime}, \omega\left(s^{\prime}, t, x\right)\right) d s^{\prime}\right) d s, \\
& \Psi(t, x)=\int_{e(t, x)}^{t} f_{2}(s, \omega(s, t, x)) \times \exp \left(\int_{r}^{t} b_{2}\left(s^{\prime}, \omega\left(s^{\prime}, t, x\right)\right) d s^{\prime}\right) d s, \\
& \Upsilon(t, x)=\int_{e(t, x)}^{t} f_{3}(s, \omega(s, t, x)) \\
& \quad \times \exp \left(\int_{r}^{t} b_{3}\left(s^{\prime}, \omega\left(s^{\prime}, t, x\right)\right) d s^{\prime}\right) d s .
\end{aligned}
$$

For $(t, x) \in R$, we have

$$
\begin{aligned}
& \Phi(t, x)=\int_{e(t, x)}^{t} f_{1}(s, \omega(s, t, x)) \\
& \times \exp \left(\int_{r}^{t} b_{1}\left(s^{\prime}, \omega\left(s^{\prime}, t, x\right)\right) d s^{\prime}\right) d s, \\
& \Psi(t, x)=\int_{e(t, x)}^{t} f_{2}(s, \omega(s, t, x)) \times \exp \left(\int_{r}^{t} b_{2}\left(s^{\prime}, \omega\left(s^{\prime}, t, x\right)\right) d s^{\prime}\right) d s, \\
& \Upsilon(t, x)=\int_{e(t, x)}^{t} f_{3}(s, \omega(s, t, x)) \\
& \quad \times \exp \left(\int_{r}^{t} b_{3}\left(s^{\prime}, \omega\left(s^{\prime}, t, x\right)\right) d s^{\prime}\right) d s .
\end{aligned}
$$

Now since $\|P(t, \cdot)\|_{L^{\infty}(0,1)} \leq 5\|\Phi(t, \cdot)\|_{L^{\infty}(0,1)},\|Q(t, \cdot)\|_{L^{\infty}(0,1)} \leq$ $5\|\Psi(t, \cdot)\|_{L^{\infty}(0,1)},\|\mathrm{H}(t, \cdot)\|_{L^{\infty}(0,1)} \leq 5\|\Upsilon(t, \cdot)\|_{L^{\infty}(0,1)}$ and $\widetilde{m}, \partial_{x} \widetilde{m}, \widetilde{n}, \partial_{x} \widetilde{n}, \widetilde{l}, \partial_{x} \widetilde{l}$ bounded, we see that for some $\lambda_{1}>0$, $\lambda_{2}>0, \lambda_{3}>0$,

$$
\begin{aligned}
& \left\|f_{1}(t, \cdot)\right\|_{L^{\infty}(0,1)} \\
& \quad \leq \lambda_{1}\left(\|\Phi(t, \cdot)\|_{L^{\infty}(0,1)}+\|\Psi(t, \cdot)\|_{L^{\infty}(0,1)}+\|\Upsilon(t, \cdot)\|_{L^{\infty}(0,1)}\right), \\
& \left\|f_{2}(t, \cdot)\right\|_{L^{\infty}(0,1)} \\
& \quad \leq \lambda_{2}\left(\|\Phi(t, \cdot)\|_{L^{\infty}(0,1)}+\|\Psi(t, \cdot)\|_{L^{\infty}(0,1)}+\|\Upsilon(t, \cdot)\|_{L^{\infty}(0,1)}\right), \\
& \left\|f_{3}(t, \cdot)\right\|_{L^{\infty}(0,1)} \\
& \quad \leq \lambda_{3}\left(\|\Phi(t, \cdot)\|_{L^{\infty}(0,1)}+\|\Psi(t, \cdot)\|_{L^{\infty}(0,1)+\|\Upsilon(t, \cdot)\|_{L^{\infty}(0,1)}}\right),
\end{aligned}
$$


since $p, q, r$, and $B_{i},(i=1,2,3)$ are bounded, we get that for some $\lambda_{1}^{\prime}>0, \lambda_{2}^{\prime}>0, \lambda_{3}^{\prime}>0$,

$$
\begin{aligned}
& \|\Phi(t, \cdot)\|_{L^{\infty}(0,1)} \\
& \qquad \lambda_{1}^{\prime} \int_{0}^{t}\left(\|\Phi(s, \cdot)\|_{L^{\infty}(0,1)}+\|\Psi(s, \cdot)\|_{L^{\infty}(0,1)}\right. \\
& \left.\quad+\|\Upsilon(s, \cdot)\|_{L^{\infty}(0,1)}\right) d s,
\end{aligned}
$$

$\|\Psi(t, \cdot)\|_{L^{\infty}(0,1)}$

$$
\begin{aligned}
\leq \lambda_{2}^{\prime} \int_{0}^{t}\left(\|\Phi(s, \cdot)\|_{L^{\infty}(0,1)}+\|\Psi(s, \cdot)\|_{L^{\infty}(0,1)}\right. \\
\left.+\|\Upsilon(s, \cdot)\|_{L^{\infty}(0,1)}\right) d s
\end{aligned}
$$

$\|\Upsilon(t, \cdot)\|_{L^{\infty}(0,1)}$

$$
\begin{gathered}
\leq \lambda_{3}^{\prime} \int_{0}^{t}\left(\|\Phi(s, \cdot)\|_{L^{\infty}(0,1)}+\|\Psi(s, \cdot)\|_{L^{\infty}(0,1)}\right. \\
\left.+\|\Upsilon(s, \cdot)\|_{L^{\infty}(0,1)}\right) d s .
\end{gathered}
$$

We can obtain that

$$
\begin{aligned}
& \|\Phi(t, \cdot)\|_{L^{\infty}(0,1)}+\|\Psi(t, \cdot)\|_{L^{\infty}(0,1)}+\|\Upsilon(t, \cdot)\|_{L^{\infty}(0,1)} \\
& \leq\left(\lambda_{1}^{\prime}+\lambda_{2}^{\prime}+\lambda_{3}^{\prime}\right) \\
& \quad \times \int_{0}^{t}\left(\|\Phi(s, \cdot)\|_{L^{\infty}(0,1)}+\|\Psi(s, \cdot)\|_{L^{\infty}(0,1)}\right. \\
& \left.\quad+\|\Upsilon(s, \cdot)\|_{L^{\infty}(0,1)}\right) d s .
\end{aligned}
$$

As a result, we get the result of the uniqueness by Gronwall's inequality when $(t, x) \in I,(t, x) \in L,(t, x) \in R$. Then, we complete the proof of the uniqueness results.

\section{Asymptotic Stabilization}

3.1. Preliminary Results. The equilibrium state that we want to stabilize is $m=n=l=0, p=q=r=G *$ $B_{i}=0$, and $i=1,2,3$. A natural idea is using Lyapunov indirection method to investigate whether the linearized system around the equilibrium state is stabilizable or not. Its stabilization would provide a local stabilization result on the nonlinear system. However, there is a difficulty in the stabilization problem. We have to prescribe $y_{l}$, and we just need to make a continuous transition at $(t, x)=(0,0)$, and that $y_{l}$ asymptotically converge in time. For convenience, the system (6)-(8) can rewrite in the following

$$
\begin{gathered}
\partial_{t} y-\breve{a}(t, x) \partial_{x} y=\breve{b}(t, x) y+\breve{f}(t, x), \\
y(0, \cdot)=y_{0}, \quad y(\cdot, 0)=y_{l}, \quad y(1, \cdot)=y_{r}, \\
\breve{g}(t, x)=G * y(t, x), \quad \breve{g}(t, 0)=\breve{g}(t, 1)=0, \\
\breve{B}(t, x)=0, \quad G * \breve{B}(t, 0)=v_{l}(t), \\
G * \breve{B}(t, 1)=v_{r}(t),
\end{gathered}
$$

where

$$
\begin{aligned}
& \breve{b}(t, x)=\left(\begin{array}{c}
2(p+G * \breve{B})_{x}+v_{x}+w_{x} \\
0 \\
0
\end{array}\right. \\
& 2(q+G * \stackrel{0}{\breve{B}})_{x}+u_{x}+w_{x} \\
& \left.02(r+G * \breve{B})_{x}+u_{x}+v_{x}\right) \text {, } \\
& \breve{a}(t, x)=\left(\begin{array}{c}
(p+G * \breve{B}+v+w) \\
0 \\
0
\end{array}\right. \\
& \left.\begin{array}{cc}
0 & 0 \\
(u+q+G * \breve{B}+w) & 0 \\
0 & (u+v+r+G * \breve{B})
\end{array}\right) \\
& y=\left(\begin{array}{c}
m \\
n \\
l
\end{array}\right), \quad y_{0}=\left(\begin{array}{c}
m_{0} \\
n_{0} \\
l_{0}
\end{array}\right) \\
& \breve{f}(t, x)=\left(\begin{array}{l}
n v_{x}+l w_{x} \\
m u_{x}+l w_{x} \\
m u_{x}+n v_{x}
\end{array}\right)=\left(\begin{array}{ccc}
0 & n & l \\
m & 0 & l \\
m & n & 0
\end{array}\right)\left(\begin{array}{c}
u_{x} \\
v_{x} \\
w_{x}
\end{array}\right), \\
& \breve{g}(t, x)=\left(\begin{array}{c}
p \\
q \\
r
\end{array}\right), \quad y_{l}=\left(\begin{array}{c}
m_{l} \\
n_{l} \\
l_{l}
\end{array}\right), \quad y_{r}=\left(\begin{array}{c}
m_{r} \\
n_{r} \\
l_{r}
\end{array}\right) .
\end{aligned}
$$

Our feedback law for (3) reads

$$
y \in C^{0}([0,1]) \longmapsto\left\{\begin{array}{l}
v_{l}(y)=A_{l}\|y\|_{C^{0}([0,1])} \\
v_{r}(y)=A_{r}\|y\|_{C^{0}([0,1])} \\
\partial_{t} y_{l}=M y_{l},
\end{array}\right.
$$

where $A_{l}>2 \sinh (1), A_{r}>A_{l} \cosh (1)+\sinh (2), M>0$, $T>0$, and $M=\left(\begin{array}{ccc}\mu & 0 & 0 \\ 0 & \mu & 0 \\ 0 & 0 & \mu\end{array}\right), \mu<0$, a symmetric matrix, is the unique matrix solution to the matrix function:

$$
P M+M^{T} P=-Z,
$$

for some symmetric positive-definite matrices $P$ and $Z$. Indeed, let $V\left(t, y_{l}\right)=y_{l}^{T} P y_{l}$ be the Lyapunov candidate, and that $y_{l}$ asymptotically converges in time is equivalent to that the time derivative of the $V, \dot{V}\left(t, y_{l}\right)=-y_{l}^{T} Z y_{l}$ is strictly negative. A fixed-point strategy will be used again to prove the existence of a solution to the closed-loop system, we begin by defining the domain of the operator.

Definition 13. Let $X$ be the space of $(g, N) \in C^{0}([0, T] \times$ $[0,1]) \times C^{0}([0,1])$ satisfying

(1) for all $(t, x) \in[0, T] \times[0,1], g(0, x)=y_{0}(x), g(t, 0)=$ $y_{0}(0) e^{M t}$,

(2) for all $t \in[0, T],\|g(t, \cdot)\|_{C^{0}([0,1])} \leq N(t)$, 
(3) $N$ is nonincreasing, and $N(0) \leq$ $\left\|y_{0}\right\|_{C^{0}([0,1]) \times C^{0}([0,1]) \times C^{0}([0,1])}$.

Lemma 14. The domain $X$ is nonempty, convex, bounded, and closed with respect to the uniform topology.

The proof is elementary and one notices that $\left(y_{0}(x) e^{M t},\left\|y_{0}\right\|_{C^{0}([0,1]) \times C^{0}([0,1]) \times C^{0}([0,1])} e^{M t}\right) \in X$, so $X$ is nonempty. of

Now for $(y, N) \in X$, we define $\stackrel{\vee}{g}$ and $G * \stackrel{\vee}{B}$ as the solutions

$$
\begin{gathered}
\forall(t, x) \in \Omega_{T}, \quad \stackrel{\vee}{g}(t, x)=G * y(t, x), \\
\stackrel{\vee}{g}(t, 0)=\stackrel{\vee}{g}(t, 1)=0, \quad \stackrel{\vee}{B}(t, x)=0, \\
G * \stackrel{\vee}{B}(t, 0)=A_{l} N(t), \quad G * \stackrel{\vee}{B}(t, 1)=A_{r} N(t) .
\end{gathered}
$$

One has the following exact formulas:

$$
\begin{aligned}
& \forall(t, x) \in \Omega_{T}, \\
& \stackrel{\vee}{g}(t, x)=- \int_{0}^{x} \sinh (x-\breve{x}) y(t, \breve{x}) d \breve{x} \\
&-\frac{\sinh (x)}{\sinh (1)} \int_{0}^{1} \sinh (\breve{x}-1) y(t, \breve{x}) d \breve{x}, \\
& G * \stackrel{\vee}{B}(t, x)= \frac{N(t)}{\sinh (1)}\left(A_{r} \sinh (x)+A_{l} \sinh (1-x)\right) .
\end{aligned}
$$

Therefore, we have the following inequalities:

$$
\begin{aligned}
& \forall(t, x) \in[0, T] \times[0,1], \\
& |\stackrel{\vee}{g}(t, x)| \\
& \leq 2(1+\cosh (1))\|y(t, \cdot)\|_{C^{0}([0,1]) \times C^{0}([0,1]) \times C^{0}([0,1])}, \\
& \left|\partial_{x} \stackrel{\vee}{g}(t, x)\right| \leq 2 \cosh (1)\|y(t, \cdot)\|_{C^{0}([0,1]) \times C^{0}([0,1]) \times C^{0}([0,1])}, \\
& \left|\partial_{x x}^{2} \stackrel{\vee}{g}(t, x)\right| \leq[2(\cosh (1)+1)+1] \\
& \times\|y(t, \cdot)\|_{C^{0}([0,1]) \times C^{0}([0,1]) \times C^{0}([0,1])}, \\
& \left|\partial_{x}(G * \stackrel{\vee}{B})(t, x)\right| \geq \frac{A_{r}-2 \cosh (1) A_{l}}{\sinh (1)} N(t), \\
& |G * \stackrel{\vee}{B}(t, x)| \geq A_{l} N(t) .
\end{aligned}
$$

Let $\stackrel{\vee}{c}(t, x)=G * \stackrel{\vee}{B}(t, x)+\stackrel{\vee}{g}(t, x)$, where $\stackrel{\vee}{c}(t, x)=$ $\left(\begin{array}{c}u \\ v \\ w\end{array}\right)(t, x)$, and in turn those provide

$$
\begin{aligned}
\stackrel{\vee}{c}(t, x) \leq & {\left[2(1+\cosh (1))+\frac{\cosh (1)}{\sinh (1)}\left(A_{r}+A_{l}\right)\right] } \\
& \times\|y(t, \cdot)\|_{C^{0}([0,1]) \times C^{0}([0,1]) \times C^{0}([0,1])}, \\
\partial_{x} \stackrel{\vee}{c}(t, x) \leq & \frac{\sinh (2)+2 A_{l} \cosh (1)-A_{r}}{\sinh (1)} \\
& \times\|y(t, \cdot)\|_{C^{0}([0,1]) \times C^{0}([0,1]) \times C^{0}([0,1])} \\
\partial_{x x}^{2} \stackrel{\vee}{c}(t, x) \leq & {\left[2(1+\cosh (1))+1+\frac{\cosh (1)}{\sinh (1)}\left(A_{r}+A_{l}\right)\right] } \\
& \times\|y(t, \cdot)\|_{C^{0}([0,1]) \times C^{0}([0,1]) \times C^{0}([0,1])} .
\end{aligned}
$$

Now, if $\omega$ is the flow of $\stackrel{\vee}{c}, \omega$ is $C^{1}$, and since $\stackrel{\vee}{c} \geq 0, \omega(\cdot, t, x)$ is nondecreasing. This allows us to define the entrance time and then the operator $S$ as follows. Let $e(t, x)=\min \{s \in[0, t] \mid$ $\omega(s, t, x)=0\}$.

Now, for for all $(t, x) \in[0, T] \times[0,1], S(y, N)=(\tilde{y}, \widetilde{N})$ with the following:

(1) if $x \geq \omega(t, 0,0)$,

$$
\begin{aligned}
& y(t, x) \\
& =y_{0}(\omega(0, t, x)) \exp \left(\int_{0}^{t} \stackrel{v}{b}(r, \omega(r, t, x)) d r\right) \\
& +\int_{0}^{t} \stackrel{\vee}{f}(r, \omega(r, t, x)) \\
& \quad \times \exp \left(\int_{r}^{t} \stackrel{v}{b}\left(r^{\prime}, \omega\left(r^{\prime}, t, x\right)\right) d r^{\prime}\right) d r,
\end{aligned}
$$

(2) if $x \leq \omega(t, 0,0)$,

$$
\begin{aligned}
& y(t, x) \\
& =y_{0}(0) e^{M e(t, x)} \exp \left(\int_{0}^{t} \vee v(r, \omega(r, t, x)) d r\right) \\
& +\int_{e(t, x)}^{t} \stackrel{\vee}{f}(r, \omega(r, t, x)) \\
& \quad \times \exp \left(\int_{r}^{t} \stackrel{v}{b}\left(r^{\prime}, \omega\left(r^{\prime}, t, x\right)\right) d r^{\prime}\right) d r,
\end{aligned}
$$

(3) $N(t)=\|\tilde{y}(t, \cdot)\|_{C^{0}([0,1])}$.

Lemma 15. (1) The operator $S$ maps $X$ to $X$.

(2) The family $S(X)$ is uniformly bounded and equicontinuous. 
(3) $S$ is continuous w.r.t. the uniform topology.

The proof is very similar to [10], except for the state y here is a three-component vector and the proof is omitted.

Now, we can apply Schauder's fixed point theorem to $S$ and get $(y, N)$ fixed point of $S$.

\subsection{Stabilization and Global Existence}

Theorem 16. For any $y_{0} \in C^{0}([0,1]) \times C^{0}([0,1]) \times C^{0}([0,1])$, there exists $y \in C^{0}\left(\Omega_{T}\right) \times C^{0}\left([0, T], C^{2}([0,1])\right)$ a weak solution of (39) satisfying

$$
\forall x \in[0,1] \quad y(0, x)=y_{0}(x) .
$$

Furthermore, any maximal solution of (39) and (41) is global, and if we let

$$
\begin{gathered}
k=\max \left(2(1+\cosh (1))+1+\frac{\cosh (1)}{\sinh (1)}\left(A_{r}+A_{l}\right),\right. \\
\left.\frac{\sinh (2)+2 A_{l} \cosh (1)-A_{r}}{\sinh (1)}\right), \\
\tau=\frac{1}{\|M\|_{3}} \ln \left(\frac{\|M\|_{3}^{2}}{8 k^{3}\left\|y_{0}\right\|_{C^{0}([0,1]) \times C^{0}([0,1]) \times C^{0}([0,1])}^{2}}\right),
\end{gathered}
$$

then we have

$$
\begin{aligned}
& \forall t \geq \tau \quad\|y(t, \cdot)\|_{C^{0}([0,1]) \times C^{0}([0,1]) \times C^{0}([0,1])} \\
& \quad \leq \frac{\|M\|_{3}}{2 k}\left(1+2 k\|M\|_{3}\right) e^{\|M\|_{3}(t-x)} .
\end{aligned}
$$

To finish the proof of Theorem (39), we have to prove the global existence of a maximal solution and the estimate (51).

Proof. First, we rewrite (46) as the following:

$$
\begin{gathered}
\forall(t, x) \in \Omega_{T} \quad \stackrel{\vee}{c}(t, x) \leq k\|y(t, \cdot)\|_{C^{0}([0,1])}, \\
\partial_{x} \stackrel{\vee}{c}(t, x) \leq k\|y(t, \cdot)\|_{C^{0}([0,1])}, \\
\partial_{x x} \stackrel{\vee}{c}(t, x) \leq k\|y(t, \cdot)\|_{C^{0}([0,1])},
\end{gathered}
$$

where $k=\max \left(2(1+\cosh (1))+1+(\cosh (1) / \sinh (1))\left(\mathrm{A}_{r}+\right.\right.$ $\left.\left.\mathrm{A}_{l}\right),\left(\sinh (2)+2 A_{l} \cosh (1)-A_{r}\right) / \sinh (1)\right)$.

For $y$ is the solution of the transport (39) and it satisfies

$$
\begin{aligned}
y(t, x)= & y(s, \omega(s, t, x)) \exp \left(\int_{0}^{t} \stackrel{v}{b}(r, \omega(r, t, x)) d r\right) \\
+ & \int_{0}^{t} \stackrel{\vee}{f}(r, \omega(r, t, x)) \\
& \quad \times \exp \left(\int_{r}^{t} \stackrel{\vee}{b}\left(r^{\prime}, \omega\left(r^{\prime}, t, x\right)\right) d r^{\prime}\right) d r .
\end{aligned}
$$

Combining those facts, we get for $t \geq s$ the following:

$$
\begin{aligned}
& |y(t, x)| \\
& \quad \leq|y(s, \omega(s, t, x))|\left(1+4 k^{2}|y(s, \omega(s, t, x))| t\right) \\
& \quad \times \exp \left(2 \int_{s}^{t} k\|y(r, \cdot)\|_{C^{0}([0,1]) \times C^{0}([0,1]) \times C^{0}([0,1])} d r\right) .
\end{aligned}
$$

We have also imposed $y(t, 0)=y(s, 0) e^{M(t-s)}$ and thanks to the existence theorem that a maximal solution of the closed loop system is global. To get a more precise statement, we consider all the between time $t$ and $s$, and we obtain.

For $0 \leq s \leq t$,

$\|y(t, \cdot)\|_{C^{0}([0,1]) \times C^{0}([0,1]) \times C^{0}([0,1])}$

$\leq\|y(s, \cdot)\|_{C^{0}([0,1]) \times C^{0}([0,1]) \times C^{0}([0,1])}$

$\times \max \left[e^{\|M\|_{3}(r-x)}\left(1+4 k^{2}\|y(s, \cdot)\|_{C^{0}([0,1]) \times C^{0}([0,1]) \times C^{0}([0,1])} r\right)\right]$

$\times \exp \left(2 k \int_{r}^{t}\|y(\alpha, \cdot)\|_{C^{0}([0,1]) \times C^{0}([0,1]) \times C^{0}([0,1])} d \alpha\right)$.

We define

$$
\begin{aligned}
g(r)= & {\left[e^{\|M\|_{3}(r-s)}\left(1+4 k^{2}\|y(r, \cdot)\|_{C^{0}([0,1]) \times C^{0}([0,1]) \times C^{0}([0,1])} r\right)\right] } \\
& \times \exp \left(2 k \int_{s}^{t}\|y(\alpha, \cdot)\|_{C^{0}([0,1]) \times C^{0}([0,1]) \times C^{0}([0,1])} d \alpha\right),
\end{aligned}
$$

and we set $g(r)=g_{1}(r)+g_{2}(r)$, where

$$
\begin{aligned}
g_{1}(r)= & e^{\|M\|_{3}(r-s)} \\
& \times \exp \left(2 k \int_{r}^{t}\|y(\alpha, \cdot)\|_{C^{0}([0,1]) \times C^{0}([0,1]) \times C^{0}([0,1])}\right), \\
g_{2}(r)= & e^{\|M\|_{3}(r-s)} 4 k^{2}\|y(r, \cdot)\|_{C^{0}([0,1]) \times C^{0}([0,1]) \times C^{0}([0,1])} r \\
& \times \exp \left(2 k \int_{r}^{t}\|y(\alpha, \cdot)\|_{C^{0}([0,1]) \times C^{0}([0,1]) \times C^{0}([0,1])}\right) .
\end{aligned}
$$

Then, we have

$$
\begin{aligned}
g^{\prime}(r) & \\
= & \left(\|M\|_{3}-2 k\|y(r, \cdot)\|_{C^{0}([0,1]) \times C^{0}([0,1]) \times C^{0}([0,1])}\right) g_{1}(r) \\
& +\left(\frac{1}{r}+\|M\|_{3}-2 k\|y(r, \cdot)\|_{C^{0}([0,1]) \times C^{0}([0,1]) \times C^{0}([0,1])}\right) \\
& \times g_{2}(r),
\end{aligned}
$$

as long as the quantity $\|y(r, \cdot)\|_{C^{0}([0,1])}$ is not equal to zero, it strictly decreases, so if $\left\|y_{0}\right\|_{C^{0}([0,1]) \times C^{0}([0,1]) \times C^{0}([0,1])}>$ 
$\|M\|_{3} / 2 k$, for $t$ small enough $\|y(t, \cdot)\|_{C^{0}([0,1]) \times C^{0}([0,1]) \times C^{0}([0,1])} \geq$ $\|M\|_{3} / 2 k$, and we have the following.

$$
\begin{aligned}
\| y(t, \cdot) & \|_{C^{0}([0,1]) \times C^{0}([0,1]) \times C^{0}([0,1])} \\
\leq & \left\|y_{0}\right\|_{C^{0}([0,1]) \times C^{0}([0,1]) \times C^{0}([0,1])} \\
& \times\left(1+4 k^{2}\left\|y_{0}\right\|_{C^{0}([0,1]) \times C^{0}([0,1]) \times C^{0}([0,1])} t\right) \mathrm{e}^{\|M\|_{3} t} .
\end{aligned}
$$

If we define $\tau=\left(1 /\|M\|_{3}\right) \quad \ln \left(\|M\|_{3}^{2} /\right.$ $\left.8 k^{3}\left\|y_{0}\right\|_{C^{0}([0,1]) \times C^{0}([0,1]) \times C^{0}([0,1])}^{2}\right)$, we get that

$$
\|y(\tau, \cdot)\|_{C^{0}([0,1]) \times C^{0}([0,1]) \times C^{0}([0,1])} \leq \frac{\|M\|_{3}}{2 k} .
$$

This provides $\tau \leq s \leq t$, the inequality (which was clear when $\left.\left\|y_{0}\right\|_{C^{0}([0,1]) \times C^{0}([0,1]) \times C^{0}([0,1])} \leq\|M\|_{3} / 2 k\right)$

$$
\begin{aligned}
\|y(t, \cdot)\|_{C^{0}([0,1]) \times C^{0}([0,1]) \times C^{0}([0,1])} & \\
\leq & \|y(\tau, \cdot)\|_{C^{0}([0,1]) \times C^{0}([0,1]) \times C^{0}([0,1])} \\
& \times\left(1+4 k^{2}\|y(\tau, \cdot)\|_{C^{0}([0,1]) \times C^{0}([0,1]) \times C^{0}([0,1])} t\right) \\
& \times \exp \left(2 k \int_{x}^{t}\|y(r, \cdot)\|_{C^{0}([0,1]) \times C^{0}([0,1]) \times C^{0}([0,1])} d r\right) \\
\leq & \frac{\|M\|_{3}}{2 k}\left(1+2 k\|M\|_{3}\right) e^{\|M\|_{3}(t-\mathrm{x})} .
\end{aligned}
$$

\section{Blow-Up Phenomena}

In this section, we present a result with the initial data and boundary profiles under a special condition that ensure strong solutions to following system blow-up in finite time as follows:

$$
\begin{aligned}
\partial_{t} m- & (u+v+w)(t, x) \partial_{x} m \\
= & \left(2(p+G * B)+v_{x}+w_{x}\right)(t, x) m \\
& +\left(n v_{x}+l w_{x}\right)(t, x), \\
\partial_{t} n- & (u+v+w)(t, x) \partial_{x} n \\
= & \left(2(q+G * B)+u_{x}+w_{x}\right)(t, x) n \\
& +\left(m u_{x}+l w_{x}\right)(t, x), \\
\partial_{t} l- & (u+v+w)(t, x) \partial_{x} l \\
= & \left(2(r+G * B)+u_{x}+v_{x}\right)(t, x) l \\
& +\left(m u_{x}+n v_{x}\right)(t, x), \\
m(0, \cdot)=m_{0}, \quad n(0, \cdot)=n_{0}, \quad l(0, \cdot)=l_{0}, \quad x \in[0,1], & \\
m(t, 0)=m_{l}= & m(t, 1)=m_{r}, \quad t \in[0, T], \\
n(t, 0)=n_{l}= & n(t, 1)=n_{r}, \quad t \in[0, T], \\
l(t, 0)=l_{l}= & l(t, 1)=l_{r}, \quad t \in[0, T],
\end{aligned}
$$

where $m=u-u_{x x}, n=v-v_{x x}, l=w-w_{x x}$. This imply that

$$
\begin{aligned}
& u(t, 0)=u(t, 1), \\
& v(t, 0)=v(t, 1), \\
& w(t, 0)=w(t, 1) .
\end{aligned}
$$

From Definition 3, we can also define

$$
\begin{array}{r}
\omega_{t}=-(u+v+w)(t, \omega(t, y)), \\
(t, x) \in[0, T] \times[0,1], \\
\omega(0, x) 1=x, \quad x \in[0,1] ;
\end{array}
$$

where $u, v$, and $w$ denote the solution to (62). Applying classical results in the theory of ordinary differential equations, one can obtain a result on which is crucial in studying blowup phenomena.

From (62), we obtain that

$$
\begin{gathered}
m_{t}-\partial_{x}(m u+m v+m w)=m u_{x}+n v_{x}+l w_{x}, \\
n_{t}-\partial_{x}(m u+n v+m w)=m u_{x}+n v_{x}+l w_{x}, \\
l_{t}-\partial_{x}(l u+l v+l w)=m u_{x}+n v_{x}+l w_{x} .
\end{gathered}
$$

Lemma 17. Let $u, v, w \in C\left([0, T) ; H^{s}\right) \cap C^{1}\left([0, T) ; H^{s-1}\right),(s \geq$ $2)$, then (64) has a unique solution $\omega \in C([0, T) \times[0,1])$. Moreover, the map $\omega(t, \cdot)$ is an increasing diffeomorphism with

$$
\begin{aligned}
\omega_{x}(t, x)=\exp \{ & -\int_{0}^{t}\left(u_{x}(s, \omega(s, x))\right. \\
& \left.\left.+v_{x}(s, \omega(s, x))+w_{x}(s, \omega(s, x)) d s\right)\right\}
\end{aligned}
$$

$>0$

$$
\omega_{x}(0, x)=1, \quad \forall x \in[0,1] .
$$

Proof. The proof is omitted here, one can see a similar proof in [12].

Now, we have the following lemma that the potential $m-$ $n, n-l, m-l$ with compactly supported initial datum $m_{0}-$ $n_{0}, n_{0}-l_{0}, m_{0}-l_{0}$ also has compact $x$ support as long as it exists.

Lemma 18. Assume that $u_{0}(t, x), v_{0}(t, x), w_{0}(t, x) \in H^{s} \times H^{s}$ with $s>(3 / 2),(u, v, w)$ is the corresponding solution, if $m_{0}-$ $n_{0}, n_{0}-l_{0}, m_{0}-l_{0}$ has compact support, then $m-n, n-l, m-l$ also has compact support, moreover, we can obtain that

$$
\begin{gathered}
\|m(t, \cdot)-n(t, \cdot)\| \leq e^{3 K T}\left\|m_{0}(\cdot)-n_{0}(\cdot)\right\|, \\
\|m(t, \cdot)-l(t, \cdot)\| \leq e^{3 K T}\left\|m_{0}(\cdot)-l_{0}(\cdot)\right\|, \\
\|n(t, \cdot)-l(t, \cdot)\| \leq e^{3 K T}\left\|n_{0}(\cdot)-l_{0}(\cdot)\right\| .
\end{gathered}
$$


Proof. Since

$$
\begin{aligned}
\frac{d}{d t} & \left(m(t, \omega(t, x)) \omega_{x}\right) \\
& =\left(m_{t}+m_{x} \omega_{t}\right) \omega_{x}+m \omega_{x t} \\
& =\left[m_{t}-\partial_{x}(m u+m v+m w)\right] \omega_{x} \\
& =\left(m u_{x}+n v_{x}+l w_{x}\right) \omega_{x} .
\end{aligned}
$$

Similarly,

$$
\begin{aligned}
& \frac{d}{d t}\left(n(t, \omega(t, x)) \omega_{x}\right)=\left(m u_{x}+n v_{x}+l w_{x}\right) \omega_{x} \\
& \frac{d}{d t}\left(l(t, \omega(t, x)) \omega_{x}\right)=\left(m u_{x}+n v_{x}+l w_{x}\right) \omega_{x} .
\end{aligned}
$$

So it follows that

$$
\begin{aligned}
& \frac{d}{d t}\left((m(t, \omega(t, x))-n(t, \omega(t, x))) \omega_{x}\right)=0, \\
& \frac{d}{d t}\left((m(t, \omega(t, x))-l(t, \omega(t, x))) \omega_{x}\right)=0, \\
& \frac{d}{d t}\left((n(t, \omega(t, x))-l(t, \omega(t, x))) \omega_{x}\right)=0 .
\end{aligned}
$$

We obtain

$$
\begin{gathered}
{[m(t, \omega(t, x))-n(t, \omega(t, x))] \omega_{x}=m_{0}(x)-n_{0}(x),} \\
{[m(t, \omega(t, x))-l(t, \omega(t, x))] \omega_{x}=m_{0}(x)-l_{0}(x),} \\
{[n(t, \omega(t, x))-l(t, \omega(t, x))] \omega_{x}=n_{0}(x)-l_{0}(x) .}
\end{gathered}
$$

From Lemma 17, we have

$$
\begin{aligned}
\omega_{x}=\exp \left(-\int_{0}^{t}\right. & u_{x}(s, \omega(s, x)) \\
& \left.+v_{x}(s, \omega(s, x))+w_{x}(s, \omega(s, x)) d s\right) .
\end{aligned}
$$

If there exist four constants $K, K_{1}, K_{2}$, and $K_{3}$ such that $u_{x} \leq$ $K_{1}, v_{x} \leq K_{2}, w_{x} \leq K_{3}$, and $K=\max \left(K_{1}, K_{2}, K_{3}\right)$, we can get that

$$
\begin{aligned}
& \|m(t, \cdot)-n(t, \cdot)\|_{L^{\infty}} \\
& =\|m(t, \omega(t, \cdot))-n(t, \omega(t, \cdot))\|_{L^{\infty}} \\
& =\| \exp \left(\int_{0}^{t} u_{x}(s, \omega(s, x))+v_{x}(s, \omega(s, x))\right. \\
& \left.\quad+w_{x}(s, \omega(s, x)) d s\right) \times\left(m_{0}(x)-n_{0}(x)\right) d s \|_{L^{\infty}} \\
& \leq e^{3 K T}\left\|m_{0}(\cdot)-n_{0}(\cdot)\right\| .
\end{aligned}
$$

Similarly,

$$
\begin{gathered}
\|m(t, \cdot)-l(t, \cdot)\| \leq e^{3 K T}\left\|m_{0}(\cdot)-l_{0}(\cdot)\right\|, \\
\|n(t, \cdot)-l(t, \cdot)\| \leq e^{3 K T}\left\|n_{0}(\cdot)-l_{0}(\cdot)\right\| .
\end{gathered}
$$

Theorem 19. Let $u_{0}(x), v_{0}(x)$, and $w_{0}(x), s>3 / 2$ and $u(t, x), v(t, x), w(t, x)$ be the solution in (62) with time T. Then, $T$ is finite if and only if

$$
\lim _{t \rightarrow T} \inf \left\{\inf _{x \in[0,1]}\left[u_{x}(x, t)\right]\right\}=-\infty
$$

or

$$
\lim _{t \rightarrow T} \inf \left\{\inf _{x \in[0,1]}\left[v_{x}(x, t)\right]\right\}=-\infty
$$

or

$$
\lim _{t \rightarrow T} \inf \left\{\inf _{x \in[0,1]}\left[w_{x}(x, t)\right]\right\}=-\infty
$$

Proof. Let $u_{0}(x), v_{0}(x)$, and $w_{0}(x), s>3 / 2$ and $u(t, x), v(t, x), w(t, x)$ be the solution (62) with time $T$. We know that $u(t, 0)=u(t, 1), v(t, 0)=v(t, 1)$, and $w(t, 0)=w(t, 1)$. By the definition of $m, n$, and $l$, we have

$$
\begin{gathered}
\|m\|_{L^{2}}^{2}=\int_{0}^{1}\left(u-u_{x x}\right)^{2} d x=\int_{0}^{1}\left(u^{2}+2 u_{x}^{2}+u_{x x}^{2}\right) d x, \\
\|n\|_{L^{2}}^{2}=\int_{0}^{1}\left(v-v_{x x}\right)^{2} d x=\int_{0}^{1}\left(v^{2}+2 v_{x}^{2}+v_{x x}^{2}\right) d x, \\
\|l\|_{L^{2}}^{2}=\int_{0}^{1}\left(w-w_{x x}\right)^{2} d x=\int_{0}^{1}\left(w^{2}+2 w_{x}^{2}+w_{x x}^{2}\right) d x .
\end{gathered}
$$

Hence, $\|u\|_{H^{2}}^{2} \leq\|m\|_{L^{2}}^{2} \leq 2\|u\|_{H^{2}}^{2},\|v\|_{H^{2}}^{2} \leq\|n\|_{L^{2}}^{2} \leq$ $2\|v\|_{H^{2}}^{2},\|w\|_{H^{2}}^{2} \leq\|l\|_{L^{2}}^{2} \leq 2\|w\|_{H^{2}}^{2}$.

Multiplying the first equation by $m$, the second one by $n$, and the third one by $l$, after integration by parts and adding up the results, we see that

$$
\begin{aligned}
& \frac{1}{2} \frac{d}{d t} \int_{0}^{1}\left(m^{2}+n^{2}+l^{2}\right) d x \\
& =\int_{0}^{1}\left[\left(2 u_{x}+v_{x}+w_{x}\right) m^{2}+\left(2 v_{x}+u_{x}+w_{x}\right) n^{2}\right. \\
& +\left(2 w_{x}+u_{x}+v_{x}\right) l^{2}+\left(m m_{x}+n n_{x}+l l_{x}\right) \\
& \times(u+v+w)+m n\left(u_{x}+v_{x}\right) \\
& \left.+\operatorname{lm}\left(u_{x}+w_{x}\right)+\ln \left(v_{x}+w_{x}\right)\right] d x \\
& =\int_{0}^{1}\left[\left(2 u_{x}+v_{x}+w_{x}-\frac{1}{2} u_{x}-\frac{1}{2} v_{x}-\frac{1}{2} w_{x}\right) m^{2}\right.
\end{aligned}
$$




$$
\begin{gathered}
+\left(2 v_{x}+u_{x}+w_{x}-\frac{1}{2} u_{x}-\frac{1}{2} v_{x}-\frac{1}{2} w_{x}\right) n^{2} \\
+\left(2 w_{x}+u_{x}+v_{x}-\frac{1}{2} u_{x}-\frac{1}{2} v_{x}-\frac{1}{2} w_{x}\right) l^{2} \\
\left.+m n\left(u_{x}+v_{x}\right)+\ln \left(u_{x}+w_{x}\right) \ln \left(v_{x}+w_{x}\right)\right] d x \\
=\int_{0}^{1}\left[\left(\frac{3}{2} u_{x}+\frac{1}{2} v_{x}+\frac{1}{2} w_{x}\right) m^{2}+\left(\frac{3}{2} v_{x}+\frac{1}{2} u_{x}+\frac{1}{2} w_{x}\right) n^{2}\right. \\
+\left(\frac{3}{2} w_{x}+\frac{1}{2} u_{x}+\frac{1}{2} v_{x}\right) l^{2}+m n\left(u_{x}+v_{x}\right) \\
\left.+\operatorname{lm}\left(u_{x}+w_{x}\right)+\ln \left(v_{x}+w_{x}\right)\right] d x \\
=\int_{0}^{1}\left[\frac{1}{2}\left(u_{x}+v_{x}+w_{x}\right)\left(m^{2}+n^{2}+l^{2}\right)\right. \\
+m^{2} u_{x}+n^{2} v_{x}+l^{2} w_{x}+m n\left(u_{x}+v_{x}\right) \\
\left.+l^{2}\left(u_{x}+w_{x}\right)+\ln \left(v_{x}+w_{x}\right)\right] d x \\
\left.+\frac{1}{2} v_{x} n^{2}+\frac{1}{2} w_{x} l^{2}\right] d x \\
+\frac{1}{2}\left(v_{x}+w_{x}\right)(l+n)^{2}+\frac{1}{2} u_{x} m^{2} \\
\left(u_{x}+v_{x}\right)(m+\mathrm{n})^{2}+\frac{1}{2}\left(u_{x}+w_{x}\right)(m+l)^{2} \\
+\frac{1}{2}
\end{gathered}
$$

So, we have

$$
\begin{aligned}
& \frac{d}{d t} \int_{0}^{1}\left(m^{2}+n^{2}+l^{2}\right) d x \\
& \leq\left(K_{1}+K_{2}+K_{3}\right) \\
& \quad \times \int_{0}^{1}\left[(m+n)^{2}+(m+l)^{2}\right. \\
& \left.\quad+(l+n)^{2}+m^{2}+n^{2}+l^{2}\right] d x \\
& \leq 5\left(K_{1}+K_{2}+K_{3}\right) \int_{0}^{1}\left(m^{2}+n^{2}+l^{2}\right) d x .
\end{aligned}
$$

By Gronwall's inequality, we get

$$
\begin{aligned}
& \|u\|_{H^{2}}^{2}+\|v\|_{H^{2}}^{2}+\|w\|_{H^{2}}^{2} \\
& \quad \leq \int_{0}^{1}\left(m^{2}+n^{2}+l^{2}\right) d x \\
& \quad \leq \exp \left[5 T\left(K_{1}+K_{2}+K_{3}\right)\right]
\end{aligned}
$$

$$
\begin{aligned}
& \times \int_{0}^{1}\left(m_{0}^{2}+n_{0}^{2}+l_{0}^{2}\right) d x \\
\leq & 2 \exp \left[5 T\left(K_{1}+K_{2}+K_{3}\right)\right] \\
& \times\left(\left\|u_{0}\right\|_{H^{2}}^{2}+\left\|v_{0}\right\|_{H^{2}}^{2}+\left\|w_{0}\right\|_{H^{2}}^{2}\right),
\end{aligned}
$$

The above inequality, Soblev's embedding theorem, ensure that the solution $(u(t, \mathrm{x}), v(t, x), w(t, x))$ cannot blow up in finite time.

On the other hand, if

$$
\lim _{t \rightarrow T} \inf \left\{\inf _{x \in[0,1]}\left[u_{x}(x, t)\right]\right\}=-\infty
$$

or

$$
\lim _{t \rightarrow T} \inf \left\{\inf _{x \in[0,1]}\left[v_{x}(x, t)\right]\right\}=-\infty
$$

or

$$
\lim _{t \rightarrow T} \inf \left\{\inf _{x \in[0,1]}\left[w_{x}(x, t)\right]\right\}=-\infty,
$$

then the solution will blow up in finite time.

\section{Acknowledgments}

The paper is supported by the National Nature Science Foundation of China (nos. 71073072 and 11171135) and the National Nature Science Foundation of Jiangsu (no. BK 2010329) and a project funded by the Priority Academic Program Development of Jiangsu Higher Education Institutions.

\section{References}

[1] R. S. Johnson, "Camassa-Holm, Korteweg-de Vries and related models for water waves," Journal of Fluid Mechanics, vol. 455, pp. 63-82, 2002.

[2] K.-H. Kwek, H. Gao, W. Zhang, and C. Qu, "An initial boundary value problem of Camassa-Holm equation," Journal of Mathematical Physics, vol. 41, no. 12, pp. 8279-8285, 2000.

[3] S. Ma and S. Ding, "On the initial boundary value problem for a shallow water equation," Journal of Mathematical Physics, vol. 45, no. 9, pp. 3479-3497, 2004.

[4] L. Molinet, "On well-posedness results for Camassa-Holm equation on the line: a survey," Journal of Nonlinear Mathematical Physics, vol. 11, no. 4, pp. 521-533, 2004.

[5] G. Rodríguez-Blanco, "On the Cauchy problem for the Camassa-Holm equation," Nonlinear Analysis. Theory, Methods \& Applications A, vol. 46, no. 3, pp. 309-327, 2001

[6] E. Wahln, "Global existence of weak solutions to the CamassaHolm equation," Communications on Pure and Applied Mathematics, vol. 21, pp. 883-906, 2006.

[7] Z. Xin and P. Zhang, "On the weak solutions to a shallow water equation," Communications on Pure and Applied Mathematics, vol. 53, no. 11, pp. 1411-1433, 2000.

[8] G. M. Coclite, H. Holden, and K. H. Karlsen, "Global weak solutions to a generalized hyperelastic-rod wave equation," SIAM Journal on Mathematical Analysis, vol. 37, no. 4, pp. 1044-1069, 2005. 
[9] J. Escher and Z. Yin, "Initial boundary value problems for nonlinear dispersive wave equations," Journal of Functional Analysis, vol. 256, no. 2, pp. 479-508, 2009.

[10] G. Zhang, Z. Qiao, and F. Liu, "Cusp and smooth solitons of the Camassa-Holm equation under an inhomogeneous boundary condition," Pacific Journal of Applied Mathematics, vol. 1, no. 1, pp. 105-121, 2008.

[11] V. Perrollaz, "Initial boundary value problem and asymptotic stabilization of the Camassa-Holm equation on an interval," Journal of Functional Analysis, vol. 259, no. 9, pp. 2333-2365, 2010.

[12] J. Escher and Z. Yin, "Initial boundary value problems of the Camassa-Holm equation," Communications in Partial Differential Equations, vol. 33, no. 1-3, pp. 377-395, 2008.

[13] G. MisioThlek, "Classical solutions of the periodic CamassaHolm equation," Geometric and Functional Analysis, vol. 12, no. 5, pp. 1080-1104, 2002.

[14] J. Zhou and L. Tian, "Blow-up of solution of an initial boundary value problem for a generalized Camassa-Holm equation," Physics Letters A, vol. 372, no. 20, pp. 3659-3666, 2008.

[15] A. Bressan and A. Constantin, "Global conservative solutions of the Camassa-Holm equation," Archive for Rational Mechanics and Analysis, vol. 183, no. 2, pp. 215-239, 2007.

[16] Y. Fu and C. Qu, "Well posedness and blow-up solution for a new coupled Camassa-Holm equations with peakons," Journal of Mathematical Physics, vol. 50, no. 1, Article ID 012906, 25 pages, 2009.

[17] Q. Hu, L. Lin, and J. Jin, "Initial boundary value problem for a coupled Camassa-Holm system with peakons," Journal of Applied Analysis, pp. 1-17, 2012.

[18] A. Constantin and R. I. Ivanov, "On an integrable two-component Camassa-Holm shallow water system," Physics Letters A, vol. 372, no. 48, pp. 7129-7132, 2008.

[19] M. Chen, S.-Q. Liu, and Y. Zhang, "A two-component generalization of the Camassa-Holm equation and its solutions," Letters in Mathematical Physics A, vol. 75, no. 1, pp. 1-15, 2006.

[20] R. I. Ivanov, "Extended Camassa-Holm hierarchy and conserved quantities," Zeitschrift Für Naturforschung, vol. 61, pp. 133-138, 2006.

[21] J. Escher, O. Lechtenfeld, and Z. Yin, "Well-posedness and blowup phenomena for the 2-component Camassa-Holm equation," Discrete and Continuous Dynamical Systems A, vol. 19, no. 3, pp. 493-513, 2007.

[22] P. Zhang and Y. Liu, "Stability of solitary waves and wavebreaking phenomena for the two-component Camassa-Holm system," International Mathematics Research Notices, no. 11, pp. 1981-2021, 2010.

[23] A. Constantin and R. I. Ivanov, "On an integrable twocomponent Camassa-Holm shallow water system," Physics Letters A, vol. 372, no. 48, pp. 7129-7132, 2008.

[24] Y. Fu, Y. Liu, and C. Qu, "Well-posedness and blow-up solution for a modified two-component periodic Camassa-Holm system with peakons," Mathematische Annalen, vol. 348, no. 2, pp. 415448, 2010.

[25] M. Zhu, "Blow-up, global existence and persistence properties for the coupled Camassa-Holm equations," Mathematical Physics, vol. 14, no. 3, pp. 197-209, 2011.

[26] J. Yin, L. Tian, and X. Fan, "Stability of negative solitary waves for an integrable modified Camassa-Holm equation," Journal of Mathematical Physics, vol. 51, no. 5, Article ID 053515, 6 pages, 2010.
[27] W. Yan, L. Tian, and M. Zhu, "Local well-posedness and blowup phenomenon for a modified two-component CamassaHolm system in Besov spaces," International Journal of Nonlinear Science, vol. 13, no. 1, pp. 99-104, 2012.

[28] P. Zhang, "Global existence of solutions to the modified Camassa-Holm shallow water equation," International Journal of Nonlinear Science, vol. 9, no. 1, pp. 123-128, 2010.

[29] L. Tian, Y. Wang, and J. Zhou, "Global conservative and dissipative solutions of a coupled Camassa-Holm equations," Journal of Mathematical Physics, vol. 52, no. 6, Article ID 063702, 29 pages, 2011.

[30] L. Tian, W. Yan, and G. Gui, "On the local well posedness and blow-up solution of a coupled Camassa-Holm equations in Besov spaces," Journal of Mathematical Physics, vol. 53, no. 1, Article ID 013701, 10 pages, 2012.

[31] Q. Hu and Z. Yin, "Well-posedness and blow-up phenomena for a periodic two-component Camassa-Holm equation," Proceedings of the Royal Society of Edinburgh A, vol. 141, no. 1, pp. 93-107, 2011.

[32] C. Guan and Z. Yin, "Global existence and blow-up phenomena for an integrable two-component Camassa-Holm shallow water system," Journal of Differential Equations, vol. 248, no. 8, pp. 2003-2014, 2010.

[33] L. Tian and Y. Xu, "Attractor for a viscous coupled CamassaHolm equation," Advances in Difference Equations, vol. 2010, Article ID 512812, 30 pages, 2010.

[34] Y. Fu and C. Qu, "On a new Three-Component Camassa-Holm equation with peakons," Communications in Theoretical Physics, vol. 53, no. 2, p. 223, 2010.

[35] Q. Hu, L. Lin, and J. Jin, "Well-posedness and blowup phenomena for a three-component Camassa-Holm system with peakons," Journal of Hyperbolic Differential Equations, vol. 9, no. 3, pp. 451-467, 2012. 


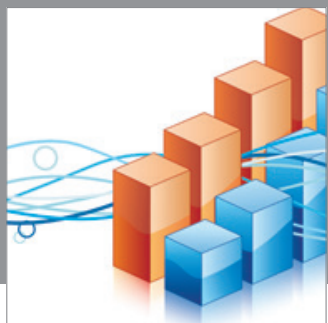

Advances in

Operations Research

mansans

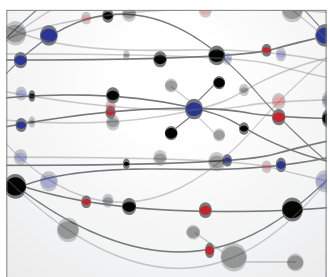

The Scientific World Journal
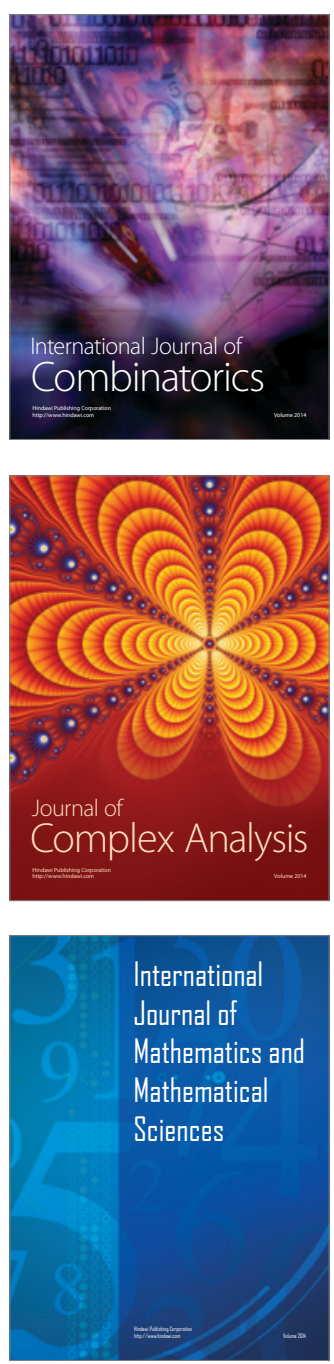
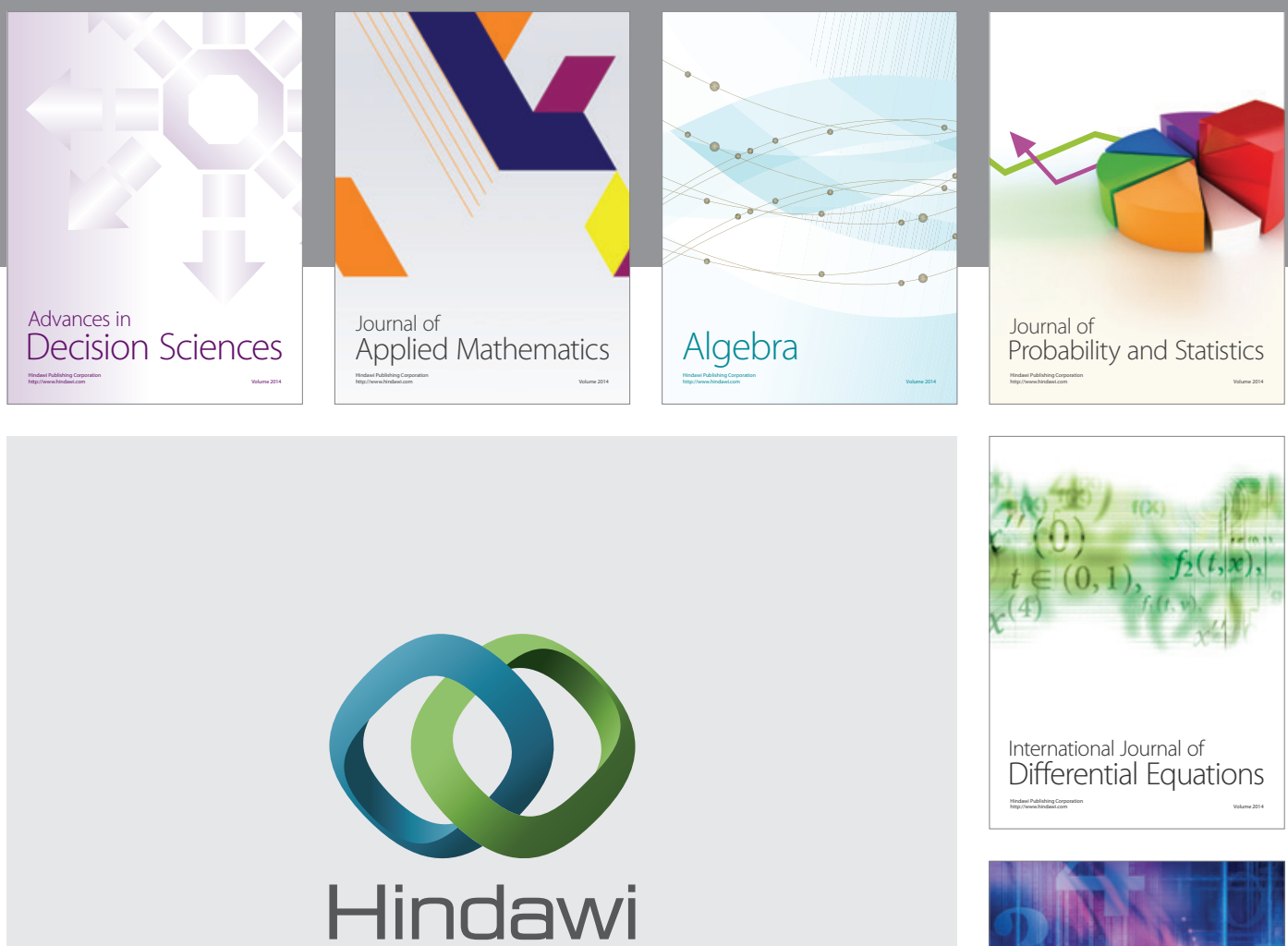

Submit your manuscripts at http://www.hindawi.com
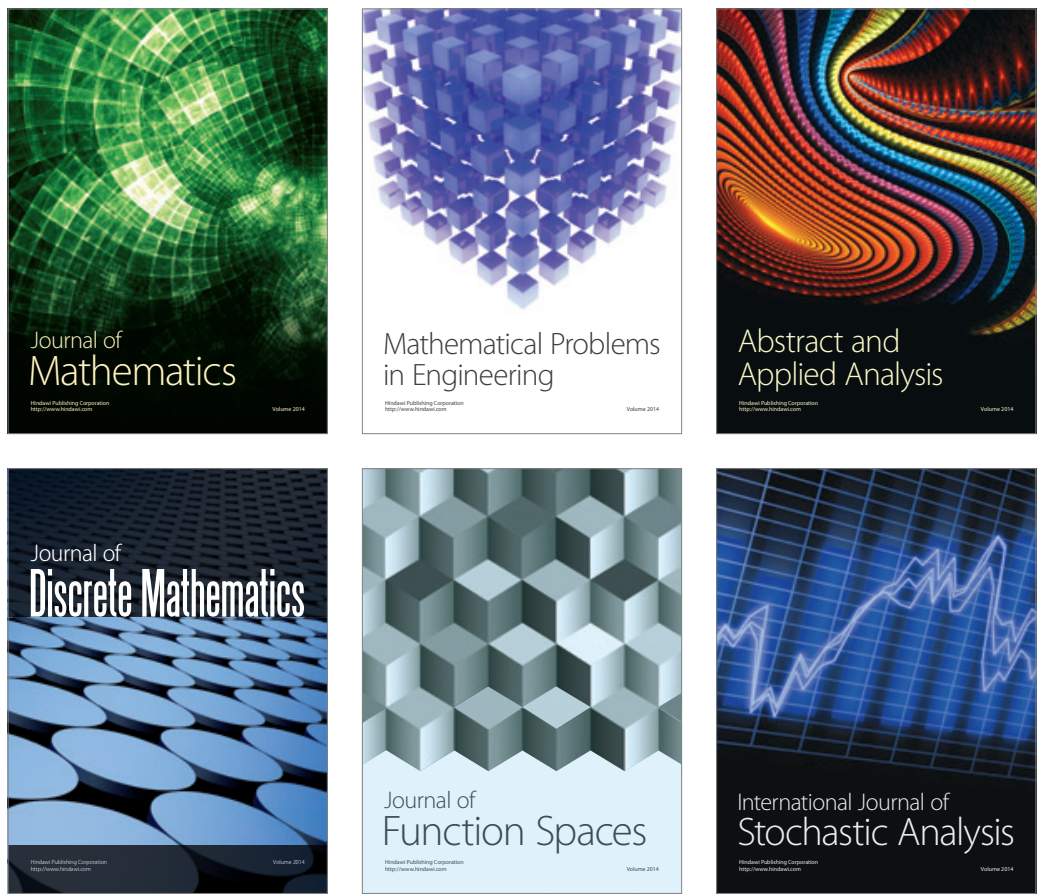

Journal of

Function Spaces

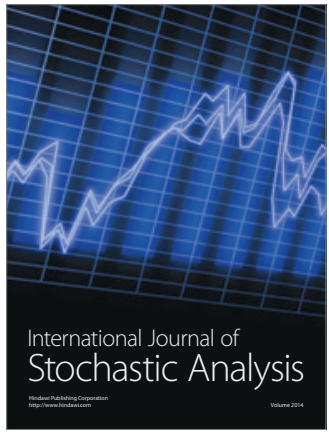

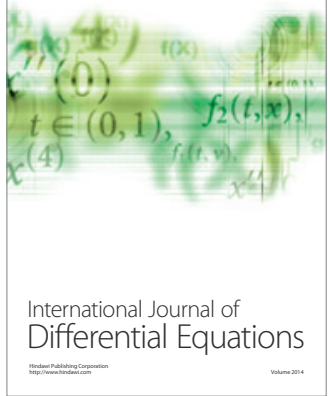
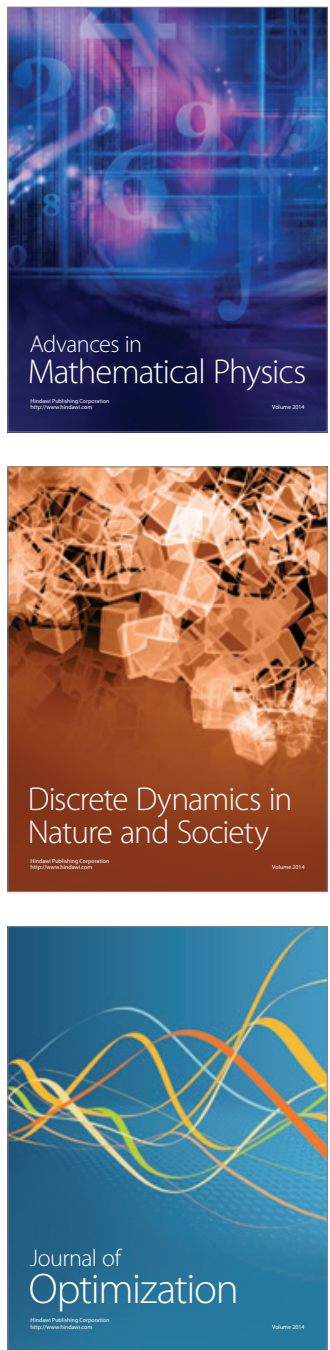